\title{
Systemic immune-inflammation index as a useful prognostic indicator predicts survival in patients with advanced gastric cancer treated with neoadjuvant chemotherapy
}

This article was published in the following Dove Press journal:

Cancer Management and Research

\section{Li Chen ${ }^{1, *}$ \\ Ying Yan ${ }^{2, *}$ \\ Lihua Zhu ${ }^{3}$ \\ Xiliang Cong' \\ Sen $\mathrm{Li}^{\prime}$ \\ Shubin Song' \\ Hongjiang Song' \\ Yingwei Xue' \\ 'Department of Gastrointestinal Surgery, Harbin Medical University Cancer Hospital, Harbin Medical University, Harbin, Heilongjiang, ${ }^{2}$ Department of Internal Oncology, Harbin The First Hospital, Harbin, Heilongjiang, ${ }^{3}$ Department of Pathogen Biology, School of Basic Medical Sciences, North China University of Science and Technology, \\ Tangshan, Hebei, China \\ *These authors contributed equally to this work}

Correspondence: Hongjiang Song Department of Gastrointestinal Surgery, Harbin Medical University Cancer Hospital, Harbin Medical University, No.I50 Haping Road, Nangang District, Harbin, Heilongjiang, I5008I, China Tel +86 I77 4564 I49I

Email hongjiangsong20I6@163.com

Lihua Zhu

Department of Pathogen Biology, School of Basic Medical Sciences, North China University of Science and Technology, No.2I Bohai Road, Caofeidian New Town, Tangshan, Hebei, 063210, China

Tel +86 I39 32554697

Email zhulihua1972@।63.com
Background and objective: A novel systemic immune-inflammation index named SII $(\mathrm{SII}=\mathrm{N} \times \mathrm{P} / \mathrm{L})$, which is based on neutrophil $(\mathrm{N})$, platelet $(\mathrm{P})$ and lymphocyte $(\mathrm{L})$ counts, has emerged and reflects comprehensively the balance of host inflammatory and immune status. We aimed to evaluate the potential prognostic significance of SII in patients with advanced gastric cancer who received neoadjuvant chemotherapy.

Subjects and methods: The retrospective analysis included data from 107 patients with advanced gastric cancer undergoing neoadjuvant chemotherapy and 185 patients with pathology-proven gastric cancer. The optimal cutoff value of SII by receiver operating characteristic curve stratified patients into low SII $\left(<600 \times 10^{9} / \mathrm{L}\right)$ and high SII $\left(\mathrm{SII} \geq 600 \times 10^{9} / \mathrm{L}\right)$ groups. The clinical outcomes of disease-free survival (DFS) and overall survival (OS) were calculated by Kaplan-Meier survival curves and compared using log-rank test. Univariate and multivariate Cox proportional hazards regression models were used to analyze the prognostic value of SII. Results: The results indicated that SII had prognostic significance using the cutoff value of $600 \times 10^{9} / \mathrm{L}$ on DFS and OS in univariate and multivariate Cox regression survival analyses. Low SII was associated with prolonged DFS and OS, and the mean DFS and OS for patients with low SII were longer than for those with high SII (57.22 vs 41.56 months and 62.25 vs 45.60 months, respectively). Furthermore, we found that patients with low SII had better 1-, 3- and 5-year rates of DFS and OS than those with high SII. In addition, patients with low SII were likely to receive DFS and OS benefits from neoadjuvant chemotherapy and postoperative chemotherapy. Conclusion: SII may qualify as a noninvasive, cost-effective, convenient and reproducible prognostic indicator for patients with advanced gastric cancer undergoing neoadjuvant chemotherapy. It may help clinicians to identify those patients who will benefit from treatment strategy decisions.

Keywords: neoadjuvant chemotherapy, gastric cancer, systemic immune-inflammation index, SII, prognosis

\section{Introduction}

Gastric cancer is one of the common malignant tumors and the second leading major cause of cancer-related morbidity and mortality all over the world. ${ }^{1}$ Although the incidence of gastric cancer has declined in recent decades, the number of gastric cancer survivors continues to grow. ${ }^{2,3}$ Every year, 738,000 people are dying from this disease, of which about 221,478 deaths are from China, accounting for nearly half of the world's gastric cancer deaths. ${ }^{4}$ In China, as a result of late diagnosis and clinically 
silent nature of the disease, many patients are diagnosed with advanced gastric carcinoma, and patients with early stage account for about $10 \%$, with an average 5-year survival rate of $10 \%-30 \%{ }^{5}$ Many studies have indicated that surgery combined with adjuvant chemoradiotherapy could improve patients' survival with early detection and treatment advances. ${ }^{6-9}$

Neoadjuvant chemotherapy has been proven to benefit patients with gastric cancer for the past several decades. The application of neoadjuvant chemotherapy in gastric cancer has received increased attention as a result of decreasing TNM stage and increasing R0 surgical resection rate, without increasing surgical morbidity and mortality compared with undergoing surgical treatment alone. ${ }^{10}$ Many of the neoadjuvant chemotherapy regimens are used for gastric cancer treatment, and the SOX (S-1 and oxaliplatin) and XELOX (oxaliplatin and capecitabine) regimens are the major first-line regimens for gastric cancer chemotherapy. Thus, it is important to find some accurate and sensitive tumor indicators of gastric cancer to improve the survival outcome. ${ }^{11}$

Some immunologic and histologic biomarkers have been used to evaluate the prognosis of gastric cancer; however, these biomarkers mainly depend on the primary tumor sample and are usually costly and time consuming. ${ }^{12}$ Cancer-related inflammation is an essential component of the tumor microenvironment, and the inflammatory cells may play a critical role in tumor development and progression. ${ }^{13}$ The routine immune and inflammatory cells, such as white blood cells, neutrophils, lymphocytes, monocytes and platelets, are detected in systemic circulation; also, neutrophil to lymphocyte ratio (NLR), monocyte to lymphocyte ratio (MLR) and platelet to lymphocyte ratio (PLR) may represent the systematic inflammatory response indicators, which have been widely proposed as prognostic factors for many malignancies. ${ }^{14-16}$

A novel systemic immune-inflammation index named SII ( $\mathrm{SII}=\mathrm{N} \times \mathrm{P} / \mathrm{L})$, which is based on neutrophil $(\mathrm{N})$, platelet (P) and lymphocyte (L) counts, has emerged; it has been reported to be associated with clinical outcomes and has proved to be a promising prognostic indicator in several solid tumors. ${ }^{17-19}$ This integrated indicator may comprehensively reflect the balance of host immune and inflammatory status. However, the SII was reported rarely in patients with gastric cancer undergoing neoadjuvant chemotherapy. This study aimed to evaluate the prognostic significance of SII in patients with gastric cancer receiving neoadjuvant chemotherapy.

\section{Subjects and methods Patient selection}

The retrospective analysis included data from 107 patients with advanced gastric cancer undergoing neoadjuvant chemotherapy at the Harbin Medical University Cancer Hospital in China between July 2007 and September 2015, and they formed the neoadjuvant chemotherapy group (NCT group). As control, we also enrolled 185 patients with pathologyproven gastric cancer who were diagnosed and had undergone potential curative resection at the Harbin Medical University Cancer Hospital in China between January 2007 and November 2009; they formed the non-neoadjuvant chemotherapy group (NNCT group). All cases were confirmed in accordance with the pathologic evidence, and the treatment details of all patients were obtained from the patients' history. This study was approved by the ethics committee of Harbin Medical University Cancer Hospital, and it complied with the standards of the Declaration of Helsinki and its later amendments. Prior to the research, written informed consent was obtained from all patients.

The inclusion criteria were as follows: 1) patients with gastric cancer were confirmed in accordance with pathologic evidence; 2) Eastern Cooperative Oncology Group performance status between 0 and 2 and Karnofsky performance status $\geq 80 ; 3$ ) life expectancy $\geq 3$ months; 4 ) adequate hematologic, liver and renal function and 5) complete clinical data and follow-up. The exclusion criteria were as follows: 1) patients having distant metastases; 2) those who received chemotherapy, radiotherapy and targeted therapy before treatment; 3) patients with inflammatory disease or autoimmune disease; 4) patients with serious complications, such as active bleeding, intestinal obstruction and obvious infection and 5) those who had blood product transfusion within 1 month before therapy.

\section{Chemotherapy protocols sOX regimen}

On the first day, oxaliplatin (Jiangsu Hengrui Medicine Co., Ltd., Lianyungang, China) at $130 \mathrm{mg} / \mathrm{m}^{2}$ was administered by intravenous infusion in $500 \mathrm{~mL}$ of $5 \%$ glucose over a period of $2 \mathrm{~h}$. From the 1 st day to the 14th day, S-1 (Taiho Pharmaceutical Co., Ltd., Tokyo, Japan) $60 \mathrm{mg}$ (twice daily) was administered peros (po).

\section{XELOX regimen}

On the first day, oxaliplatin $\left(130 \mathrm{mg} / \mathrm{m}^{2}\right)$ was administered by intravenous infusion in $500 \mathrm{~mL}$ of $5 \%$ glucose over a 
period of $2 \mathrm{~h}$. From the 1 st day to the 14th day, capecitabine (Shanghai Roche Pharmaceuticals Ltd., Shanghai, China) $1500 \mathrm{mg}$ (twice daily) was administered po. A cycle of the two regimens was repeated every 3 weeks.

\section{Classification standard and response evaluation}

Pathology type was classified as adenocarcinoma (tubular adenocarcinoma and papillary adenocarcinoma), mucinous carcinoma, signet ring cell carcinoma and mixed carcinoma. TNM stage system was used as per the eighth edition of the Union for International Cancer Control and the American Joint Committee on Cancer TNM stage classification. ${ }^{20}$ Lauren classification was defined as intestinal type, diffuse type and mixed type. ${ }^{21}$ Borrmann classification was categorized into five groups: Borrmann 0, Borrmann I, Borrmann II, Borrmann III and Borrmann IV. ${ }^{22}$ Response rates were determined according to the Response Evaluation Criteria In Solid Tumors guidelines, ${ }^{23}$ and included the following categories: complete response (CR), partial response (PR), stable disease (SD) and progression of disease (PD). The clinical objective response rate was defined as $\mathrm{CR}$ and $\mathrm{PR}$, and nonclinical response was defined as either SD or PD. The Clinical benefit rate was defined as CR, PR and SD. The toxicity of neoadjuvant chemotherapy was evaluated according to the National Cancer Institute Common Toxicity Criteria. ${ }^{24}$

\section{Peripheral venous blood parameters}

Peripheral venous blood samples were collected within 1 week prior to neoadjuvant chemotherapy for NCT group or surgery for NNCT group. The blood samples were collected in a sterile EDTA tube and obtained with empty stomach. Hematologic parameters were analyzed by XE-2100 hematology analyzer (Sysmex, Kobe, Japan).

\section{Follow-up}

All patients were routinely followed up as inpatients and outpatients every 3-6 months for the first to second year after surgery, every 6-12 months interval thereafter, then annually and until death. Follow-up assessments included laboratory tests, physical examination, multislice computed tomography, gastroscopy and some other examinations as it fits. Disease-free survival (DFS) was defined as the time (in months) from the date of surgery to the date of relapse (local recurrence and/or distant metastases), death from any cause or last follow-up. Overall survival (OS) was defined as the time (in months) from the date of surgery to the date of death from any cause or last follow-up. The last follow-up date was
December 10, 2016. Survival duration was measured from the date of surgery until death or last follow-up.

\section{Statistical analysis}

Statistical analyses were performed using the SPSS software (version 17.0; SPSS Inc., Chicago, IL, USA) and GraphPad prism software (version 5.0; GraphPad Inc., La Jolla, CA, USA). The optimal cutoff value was obtained using receiver operating characteristic curve (ROC) analyses. The area under the curve was used to assess the predictive value. The ratio closest to the point with maximum sensitivity and specificity was defined as the optimal cutoff value. The categorical variables in clinicopathologic database were presented as frequencies and percentages (\%) and compared using the chi-square test or Fisher's exact test. The patients' baseline characteristics were expressed as the mean \pm standard error for the qualitative variables and compared using Student's $t$-test. The clinical outcomes of DFS and OS were calculated by Kaplan-Meier survival curves and compared using logrank test. The independent prognostic factors and prognostic value of the SII were assessed by univariate and multivariate Cox proportional hazards regression model. Alpha was set at 0.05 , and a two-tailed $P<0.05$ was considered to indicate a statistically significant difference.

\section{Results}

\section{Demographic and clinicopathologic characteristics of patients}

As shown in Table 1, a total of 292 patients with gastric cancer were enrolled in this study; 107 patients were assigned to the NCT group and 185 patients to the NNCT group. ROC analysis was used to determine an optimal cutoff value of the SII $\left(600 \times 10^{9} / \mathrm{L}\right.$ was the optimum cutoff value), and this optimal cutoff value was used for all analyses. Thereafter, the patients were stratified into two groups by the optimal cutoff value of SII: a low SII group (SII $\left.<600 \times 10^{9} / \mathrm{L}\right)$ and a high SII group (SII $\geq 600 \times 10^{9} / \mathrm{L}$ ). Table 1 shows the distribution of the demographic and clinicopathologic characteristics of 292 patients in the two groups. Males and females numbered 207 and 85, respectively; the median age of all patients was 57 years, with the range being 28-77 years. There were 169 patients $(57.9 \%)$ in the low SII group and 123 patients $(42.1 \%)$ in the high SII group. In the NCT group, 52 patients (48.6\%) were in the low SII group and 55 patients $(51.4 \%)$ in the high SII group. In the NNCT group, 117 patients $(63.2 \%)$ were in the low SII group and 68 patients $(36.8 \%)$ in the high SII group. In the NCT group, a low SII was significantly associated with carcinoembryonic antigen (CEA) $\left(\chi^{2}=4.114, P=0.043\right)$. In the NNCT group, a low SII 
Table I Demographic and clinicopathologic characteristics of 292 patients with gastric cancer

\begin{tabular}{|c|c|c|c|c|c|c|c|c|}
\hline \multirow[t]{2}{*}{ Parameters } & \multicolumn{3}{|l|}{ NCT } & \multirow[t]{2}{*}{$P$-value } & \multicolumn{3}{|l|}{ NNCT } & \multirow[t]{2}{*}{$P$-value } \\
\hline & Low SII <600 & High SII $\geq 600$ & $\chi^{2}$ & & Low SII <600 & High SII $\geq 600$ & $\chi^{2}$ & \\
\hline Cases (n) & 52 & 55 & & & 117 & 68 & & \\
\hline Age (years) & & & 2.732 & 0.098 & & & 0.558 & 0.455 \\
\hline$<57$ & 31 & 24 & & & 60 & 31 & & \\
\hline$\geq 57$ & 21 & 31 & & & 57 & 37 & & \\
\hline Gender & & & 3.098 & 0.078 & & & 5.119 & 0.024 \\
\hline Male & 36 & 46 & & & 86 & 39 & & \\
\hline Female & 16 & 9 & & & 31 & 29 & & \\
\hline BMI & & & 0.753 & 0.386 & & & & \\
\hline$<22.10$ & 28 & 25 & & & & & & \\
\hline$\geq 22.10$ & 24 & 30 & & & & & & \\
\hline ABO blood type & & & $0.45 I$ & 0.930 & & & 2.880 & 0.411 \\
\hline A & 16 & 14 & & & 32 & 15 & & \\
\hline B & 17 & 19 & & & 33 & 27 & & \\
\hline O & 14 & 17 & & & 37 & 17 & & \\
\hline$A B$ & 5 & 5 & & & 15 & 9 & & \\
\hline Radical resection & & & 1.809 & 0.405 & & & 1.792 & 0.426 \\
\hline RO & 30 & 30 & & & 100 & 53 & & \\
\hline RI & 9 & 15 & & & 10 & 9 & & \\
\hline R2 & 13 & 10 & & & 7 & 6 & & \\
\hline Type of surgery & & & 0.159 & $0.924^{*}$ & & & 1.078 & 0.583 \\
\hline Distal gastrectomy & 30 & 30 & & & 79 & 42 & & \\
\hline Proximal gastrectomy & 3 & 4 & & & 10 & 5 & & \\
\hline Total gastrectomy & 19 & 21 & & & 28 & 21 & & \\
\hline Differentiation & & & 0.042 & $0.979 *$ & & & 0.417 & $0.812^{*}$ \\
\hline Poorly differentiated & 32 & 33 & & & 72 & 39 & & \\
\hline Moderately differentiated & 17 & 19 & & & 44 & 28 & & \\
\hline Well differentiated & 3 & 3 & & & I & 1 & & \\
\hline Primary tumor site & & & 3.005 & $0.223^{*}$ & & & 3.918 & 0.141 \\
\hline Upper one third & 3 & 6 & & & 12 & 5 & & \\
\hline Middle one third & 16 & 23 & & & 38 & 32 & & \\
\hline Lower one third & 33 & 26 & & & 67 & 31 & & \\
\hline Pathology & & & 4.417 & $0.353^{*}$ & & & 5.418 & $0.144 *$ \\
\hline Normal (Tis) & 7 & 2 & & & 0 & 0 & & \\
\hline Adenocarcinoma & 26 & 35 & & & 82 & 44 & & \\
\hline Mucinous carcinoma & 4 & 4 & & & 7 & 11 & & \\
\hline Signet ring cell carcinoma & 7 & 5 & & & 22 & II & & \\
\hline Mixed carcinoma & 8 & 9 & & & 6 & 2 & & \\
\hline \multicolumn{9}{|l|}{ Clinical TNM classification } \\
\hline T stage & & & 0.068 & $0.795^{*}$ & & & & \\
\hline T3 & 4 & 5 & & & & & & \\
\hline $\mathrm{T} 4$ & 48 & 50 & & & & & & \\
\hline$N$ stage & & & 5.554 & $0.062^{*}$ & & & & \\
\hline No & 14 & 16 & & & & & & \\
\hline NI & 33 & 39 & & & & & & \\
\hline N2 & 5 & 0 & & & & & & \\
\hline TNM stage & & & $1.05 \mathrm{I}$ & 0.305 & & & & \\
\hline II & 9 & 14 & & & & & & \\
\hline III & 43 & 41 & & & & & & \\
\hline \multicolumn{9}{|c|}{ Pathologic TNM classification } \\
\hline T stage & & & 5.378 & $0.25 I^{*}$ & & & 3.812 & $0.432 *$ \\
\hline Tis $+\mathrm{TI}$ & 10 & 7 & & & 3 & 3 & & \\
\hline $\mathrm{T} 2$ & 0 & 3 & & & 15 & 3 & & \\
\hline T3 & 21 & 21 & & & 23 & 14 & & \\
\hline $\mathrm{T} 4 \mathrm{a}$ & 12 & 9 & & & 67 & 42 & & \\
\hline T4b & 9 & 15 & & & 9 & 6 & & \\
\hline $\mathrm{N}$ stage & & & 6.368 & 0.173 & & & 10.480 & 0.033 \\
\hline
\end{tabular}


Table I (Continued)

\begin{tabular}{|c|c|c|c|c|c|c|c|c|}
\hline \multirow[t]{2}{*}{ Parameters } & \multicolumn{3}{|l|}{ NCT } & \multirow[t]{2}{*}{$P$-value } & \multicolumn{3}{|l|}{ NNCT } & \multirow[t]{2}{*}{$P$-value } \\
\hline & Low SII $<600$ & High SII $\geq 600$ & $\chi^{2}$ & & Low SII $<600$ & High SII $\geq 600$ & $\chi^{2}$ & \\
\hline No & 18 & 14 & & & 17 & 10 & & \\
\hline $\mathrm{NI}$ & 13 & 10 & & & 24 & 9 & & \\
\hline N2 & 5 & 13 & & & 32 & 19 & & \\
\hline N3a & 10 & 15 & & & 36 & 15 & & \\
\hline N3b & 6 & 3 & & & 8 & 15 & & \\
\hline Metastasis & & & 0.403 & $0.525^{*}$ & & & 0.031 & $0.860 *$ \\
\hline Mo & 50 & 54 & & & 113 & 66 & & \\
\hline MI & 2 & 1 & & & 4 & 2 & & \\
\hline TNM stage & & & 1.503 & $0.682^{*}$ & & & 0.136 & $0.987^{*}$ \\
\hline Tis +1 & 9 & 6 & & & 5 & 3 & & \\
\hline II & 15 & 16 & & & 21 & 11 & & \\
\hline III & 26 & 32 & & & 87 & 52 & & \\
\hline IV & 2 & 1 & & & 4 & 2 & & \\
\hline Total lymph nodes & & & 0.031 & 0.861 & & & 1.057 & 0.304 \\
\hline$<22$ & 19 & 21 & & & 71 & 36 & & \\
\hline$\geq 22$ & 33 & 34 & & & 46 & 32 & & \\
\hline Positive lymph nodes & & & 0.972 & 0.615 & & & 1.228 & $0.54 I$ \\
\hline 0 & 18 & 15 & & & 17 & 9 & & \\
\hline$<5$ & 14 & 14 & & & 39 & 18 & & \\
\hline$\geq 5$ & 20 & 26 & & & 61 & 41 & & \\
\hline CEA $(\mathrm{ng} / \mathrm{mL})$ & & & 4.114 & 0.043 & & & 0.004 & 0.950 \\
\hline$<2.72$ & 31 & 22 & & & 70 & 41 & & \\
\hline$\geq 2.72$ & 21 & 33 & & & 47 & 27 & & \\
\hline CAI9-9 (U/mL) & & & 0.231 & 0.631 & & & 3.962 & 0.047 \\
\hline$<12.12$ & 27 & 26 & & & 71 & 31 & & \\
\hline$\geq 12.12$ & 25 & 29 & & & 46 & 37 & & \\
\hline Lauren classification & & & 1.636 & $0.44 I$ & & & 4.950 & $0.084^{*}$ \\
\hline Intestinal & 26 & 31 & & & 82 & 44 & & \\
\hline Diffuse & 18 & 13 & & & 4 & 8 & & \\
\hline Mixed & 8 & II & & & 31 & 16 & & \\
\hline Borrmann classification & & & 2.860 & $0.414^{*}$ & & & 0.195 & $0.978^{*}$ \\
\hline Borrmann I & 1 & 0 & & & 6 & 3 & & \\
\hline Borrmann II & 14 & 10 & & & 15 & 10 & & \\
\hline Borrmann III & 29 & 38 & & & 83 & 47 & & \\
\hline Borrmann IV & 8 & 7 & & & 13 & 8 & & \\
\hline Tumor size $(\mathrm{mm})$ & & & 0.092 & $0.76 I$ & & & 9.329 & 0.002 \\
\hline$<50$ & 28 & 28 & & & 52 & 15 & & \\
\hline$\geq 50$ & 24 & 27 & & & 65 & 53 & & \\
\hline
\end{tabular}

Notes: *Fisher's exact test. SII is a novel systemic immune-inflammation index (SII=N $\times P / L)$, which is based on neutrophil $(N)$, platelet $(P)$ and lymphocyte $(L)$ counts. Abbreviations: BMI, body mass index; NCT, neoadjuvant chemotherapy; NNCT, non-neoadjuvant chemotherapy; CAI9-9, carbohydrate antigen 19-9; CEA, carcinoembryonic antigen.

was significantly associated with gender $\left(\chi^{2}=5.119, P=0.024\right)$, pathologic $\mathrm{N}$ stage $\left(\chi^{2}=10.480, P=0.033\right), \mathrm{CA} 19-9\left(\chi^{2}=3.962\right.$, $P=0.047)$ and tumor size $\left(\chi^{2}=9.329, P=0.002\right)$.

\section{Blood parameters}

The median white blood cell $(\mathrm{W})$, hemoglobin $(\mathrm{Hb})$, neutrophil $(\mathrm{N})$, monocyte $(\mathrm{M})$, platelet $(\mathrm{P})$ and lymphocyte $(\mathrm{L})$ counts were $6.17 \times 10^{9} / \mathrm{L}, 126 \times 10^{9} / \mathrm{L}, 3.74 \times 10^{9} / \mathrm{L}, 0.42 \times 10^{9} / \mathrm{L}$, $262 \times 10^{9} / \mathrm{L}$ and $1.74 \times 10^{9} / \mathrm{L}$, respectively. The optimum cutoff values of NLR, MLR and PLR by ROC analysis were $1.65,0.17$ and 133 , respectively. A low SII was significantly associated with white blood cell $(P<0.05), \mathrm{Hb}(P<0.01)$ neutrophils $(P<0.001)$, platelets $(P<0.001)$, NLR $(P<0.001)$, $\operatorname{MLR}(P<0.05)$ and PLR $(P<0.001)$. However, the low SII was not associated with monocyte counts in both NCT and NNCT groups $(P>0.05)$. In the NNCT group, a low SII was significantly associated with lymphocyte counts $(P<0.001$; Table 2$)$.

\section{Univariate and multivariate Cox regression survival analyses}

The median DFS and OS of the patients were 26.23 months (range 1.03-112.37 months) and 32.77 months (range 
Table 2 Correlation between SII and hematologic parameters

\begin{tabular}{|c|c|c|c|c|c|c|c|c|}
\hline \multirow[t]{2}{*}{ Parameters } & \multicolumn{3}{|l|}{ NCT } & \multirow[t]{2}{*}{$P$-value } & \multicolumn{3}{|l|}{ NNCT } & \multirow[t]{2}{*}{$P$-value } \\
\hline & Low SII $<600$ & High SII $\geq 600$ & $\chi^{2}$ & & Low SII <600 & High SII $\geq 600$ & $\chi^{2}$ & \\
\hline Cases (n) & 52 & 55 & & & 117 & 68 & & \\
\hline White blood cell (W) & & & 15.680 & $<0.001$ & & & 6.578 & 0.010 \\
\hline$<6.17$ & 33 & 14 & & & 71 & 28 & & \\
\hline$\geq 6.17$ & 19 & 41 & & & 46 & 40 & & \\
\hline $\mathrm{Hb}$ & & & 6.765 & 0.009 & & & 16.530 & $<0.001$ \\
\hline$<126$ & 24 & 39 & & & 38 & 43 & & \\
\hline$\geq 126$ & 28 & 16 & & & 79 & 25 & & \\
\hline Neutrophils (N) & & & 28.230 & $<0.001$ & & & 28.660 & $<0.001$ \\
\hline$<3.74$ & 39 & 13 & & & 77 & 17 & & \\
\hline$\geq 3.74$ & 13 & 42 & & & 40 & 51 & & \\
\hline Monocyte (M) & & & 2.058 & 0.151 & & & 1.146 & 0.284 \\
\hline$<0.42$ & 21 & 15 & & & 68 & 34 & & \\
\hline$\geq 0.42$ & 31 & 40 & & & 49 & 34 & & \\
\hline Platelet (P) & & & 19.190 & $<0.001$ & & & 18.100 & $<0.00$ I \\
\hline$<262$ & 32 & 11 & & & 79 & 24 & & \\
\hline$\geq 262$ & 20 & 44 & & & 38 & 44 & & \\
\hline Lymphocyte (L) & & & 0.448 & 0.503 & & & 17.090 & $<0.001$ \\
\hline$<1.74$ & 25 & 30 & & & 44 & 47 & & \\
\hline$\geq 1.74$ & 27 & 25 & & & 73 & 21 & & \\
\hline NLR & & & 29.340 & $<0.00 I^{*}$ & & & 44.610 & $<0.001$ \\
\hline$<1.65$ & 24 & I & & & 57 & I & & \\
\hline$\geq 1.65$ & 28 & 54 & & & 60 & 67 & & \\
\hline MLR & & & 67.501 & $<0.001$ & & & 6.600 & 0.010 \\
\hline$<0.17$ & 46 & 5 & & & 35 & 9 & & \\
\hline$\geq 0.17$ & 6 & 50 & & & 82 & 59 & & \\
\hline PLR & & & 33.400 & $<0.00 I^{*}$ & & & 47.790 & $<0.001$ \\
\hline$<133$ & 28 & 2 & & & 77 & 9 & & \\
\hline$\geq 133$ & 24 & 53 & & & 40 & 59 & & \\
\hline
\end{tabular}

Notes: *Fisher's exact test. SIl is a novel systemic immune-inflammation index (SII=N $\times P / L)$, which is based on neutrophil $(N)$, platelet $(P)$ and lymphocyte $(L)$ counts. Abbreviations: Hb, hemoglobin; MLR, monocyte to lymphocyte ratio; NCT, neoadjuvant chemotherapy; NLR, neutrophil to lymphocyte ratio; NNCT, non-neoadjuvant chemotherapy; PLR, platelet to lymphocyte ratio.

3.03-117.37 months), respectively (Figure 1A, B). In univariate analysis, radical resection, pathologic $\mathrm{T}$ stage, pathologic $\mathrm{N}$ stage, pathologic $\mathrm{M}$ stage, pathologic TNM stage, total lymph nodes, positive lymph nodes, Borrmann classification, lymphocyte counts and SII were the significant prognostic factors for DFS and OS. The results of multivariate Cox regression analysis showed the factors associated with DFS and $\mathrm{OS}$ to be radical resection, pathologic $\mathrm{T}$ stage, pathologic $\mathrm{N}$ stage, pathologic M stage, pathologic TNM stage, total lymph nodes, positive lymph nodes, Borrmann classification, lymphocyte counts and SII (Table 3).

The results indicated that SII had prognostic significance for DFS and OS using the cutoff value of $600 \times 10^{9} / \mathrm{L}$. In univariate analysis, low SII was associated with prolonged DFS and OS ( $P=0.020$, hazard ratio [HR]: $1.607,95 \% \mathrm{CI}$ : $1.076-2.398$ and $P=0.028$, HR: $1.574,95 \%$ CI: $1.049-2.361$, respectively). In multivariate Cox regression analysis, low SII was associated with prolonged DFS and OS $(P=0.003$,
HR: $1.574,95 \%$ CI: $1.161-2.134$ and $P=0.002$, HR: 1.637 , 95\% CI: 1.194-2.245, respectively; Table 3). Among 292 patients, the mean DFS and OS for patients with low SII were 57.22 months (range 1.93-112.37 months) and 62.25 months (range 3.03-117.37 months), respectively, and the mean DFS and OS for patients with high SII were 41.56 months (range 1.03-99.50 months) and 45.60 months (range 3.10-104.10 months), respectively. By using log-rank test, the mean DFS and OS time for patients with low SII were longer than for those with high SII $\left(\chi^{2}=4.428, P=0.035\right.$ and $\chi^{2}=5.569, P=0.018$, respectively; Figure 1C, D).

We further studied the differences with SII in the NCT and NNCT groups. In the NCT group, the mean DFS and OS for patients with low SII were 55.57 months (range 4.90-112.37 months) and 57.52 months (range 4.90-112.37 months), respectively. The mean DFS and OS for patients with high SII were 32.31 months (range 1.17-96.00 months) and 34.60 months (range 4.03-96.00 months), respectively. By using 

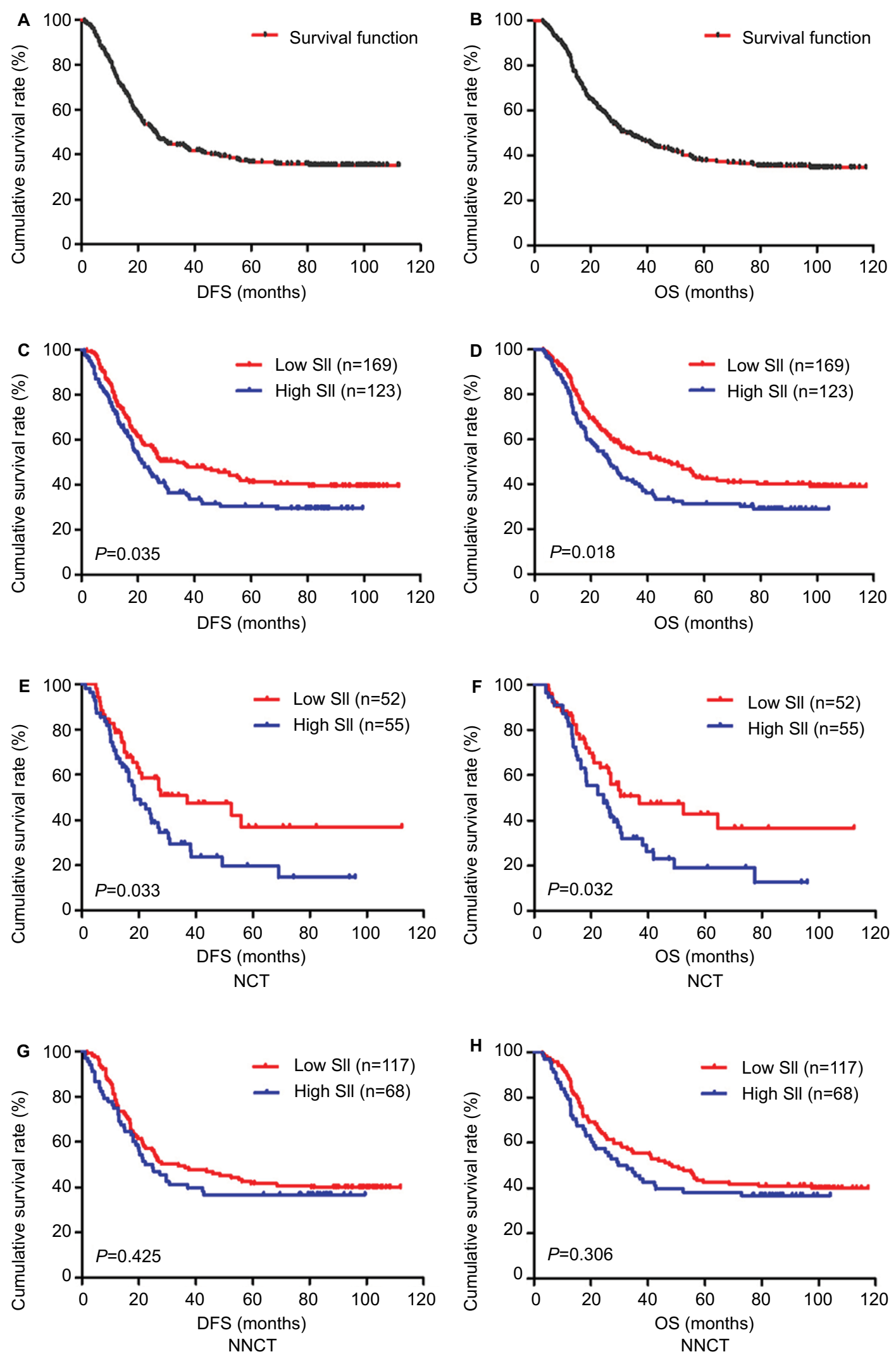

Figure I DFS and OS of patients with gastric cancer.

Notes: (A) Kaplan-Meier analysis of DFS of all patients with gastric cancer. (B) Kaplan-Meier analysis of OS of all patients with gastric cancer. (C) Kaplan-Meier analysis of DFS for the SIl of all patients with gastric cancer. (D) Kaplan-Meier analysis of OS for the SIl of all patients with gastric cancer. (E) Kaplan-Meier analysis of DFS for the SII of patients with gastric cancer in the NCT group. (F) Kaplan-Meier analysis of OS for the Sll of patients with gastric cancer in the NCT group. (G) Kaplan-Meier analysis of DFS for the SIl of patients with gastric cancer in the NNCT group. (H) Kaplan-Meier analysis of OS for the SIl of patients with gastric cancer in the NNCT group. SII is a novel systemic immune-inflammation index (SIl=N $\times P / L)$, which is based on neutrophil $(\mathrm{N})$ ), platelet $(\mathrm{P})$ and lymphocyte $(\mathrm{L})$ counts.

Abbreviations: DFS, disease-free survival; NCT, neoadjuvant chemotherapy; NNCT, non-neoadjuvant chemotherapy; OS, overall survival. 


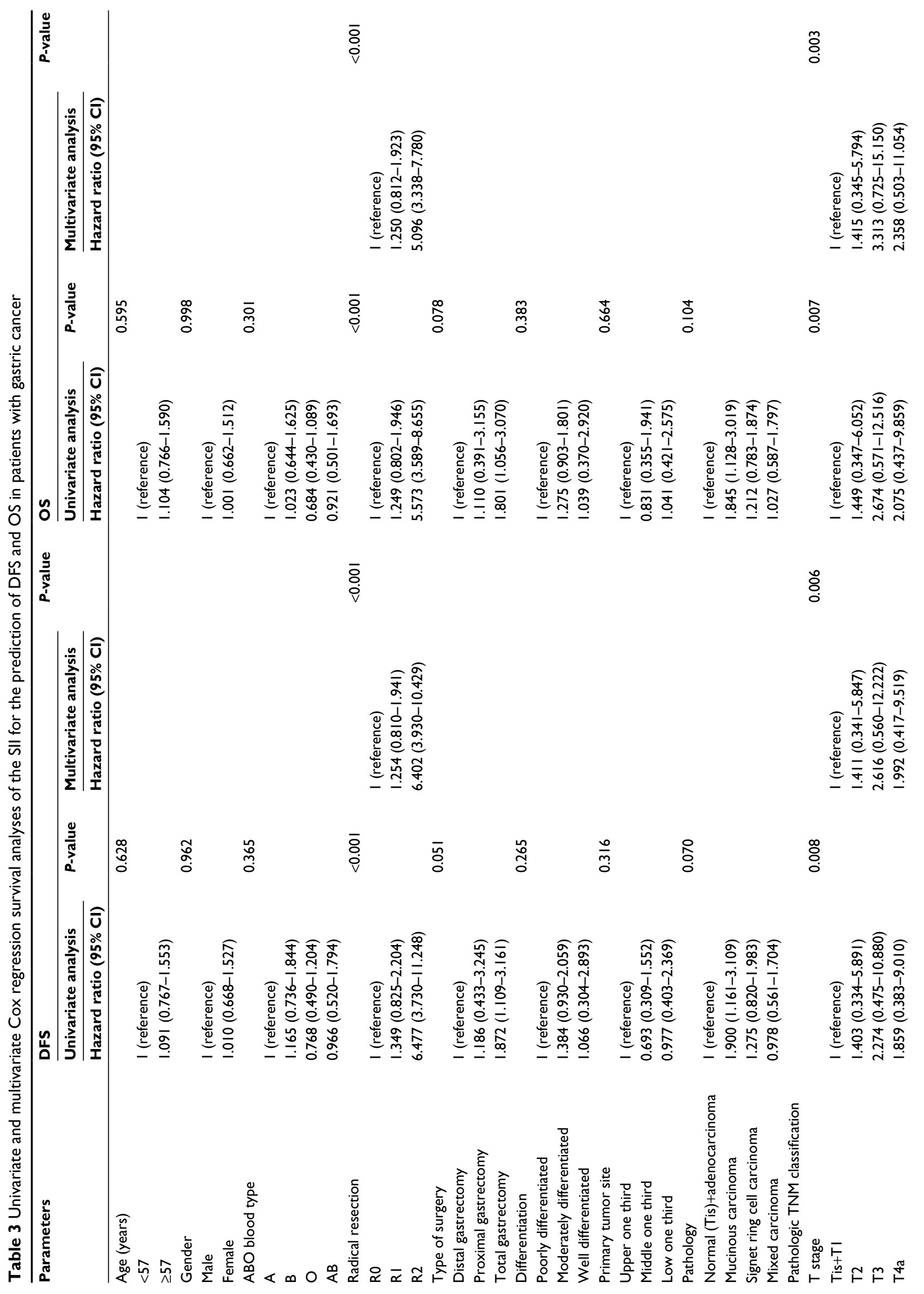




\section{$\begin{array}{lllll}\bar{o} & \bar{o} & \bar{o} & \bar{o} & \bar{o} \\ \dot{i} & \bar{i} & \bar{v} & \bar{o} & \end{array}$}

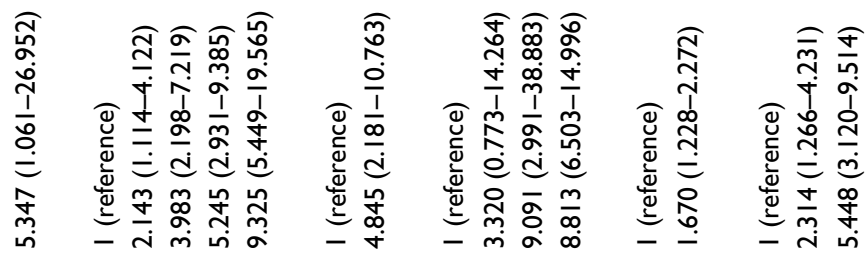

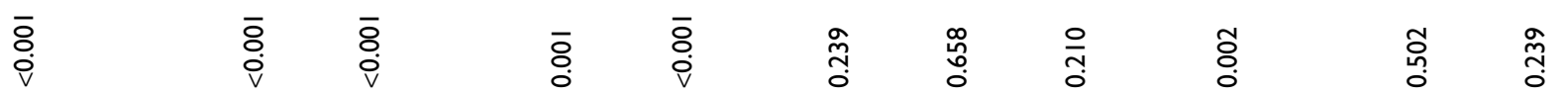

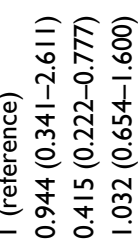

$\stackrel{\circ}{\circ}$

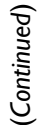

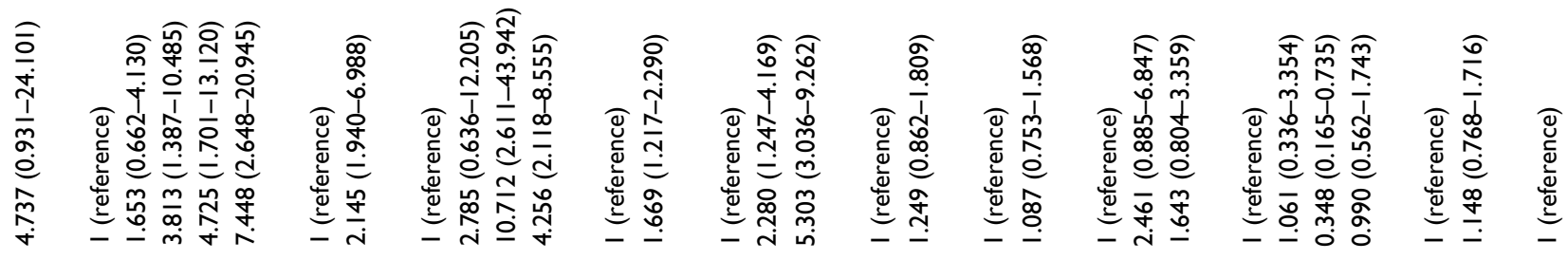
$\begin{array}{lllll}\bar{o} & \bar{o} & \bar{o} & \stackrel{0}{0} & \overline{0} \\ \dot{0} & \bar{v} & \bar{v} & o & \bar{v}\end{array}$
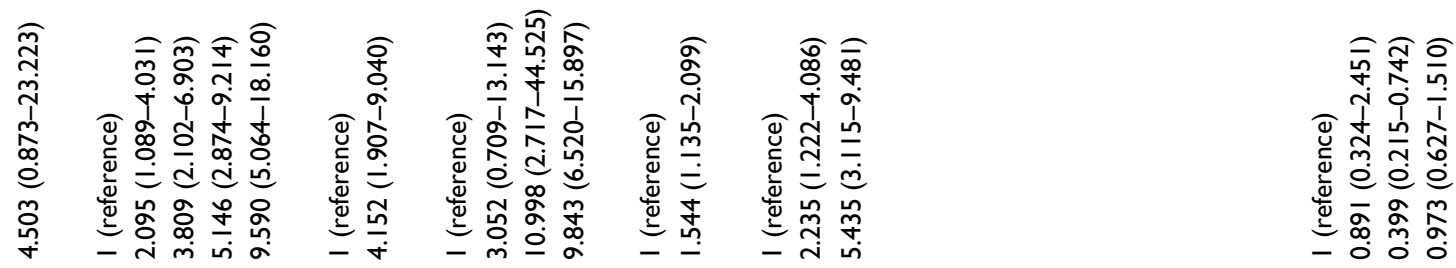

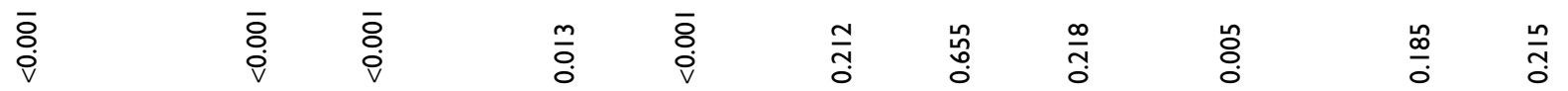

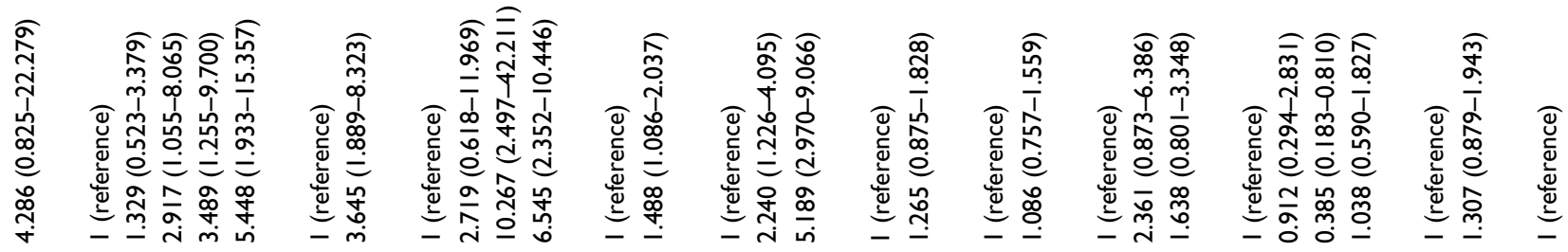

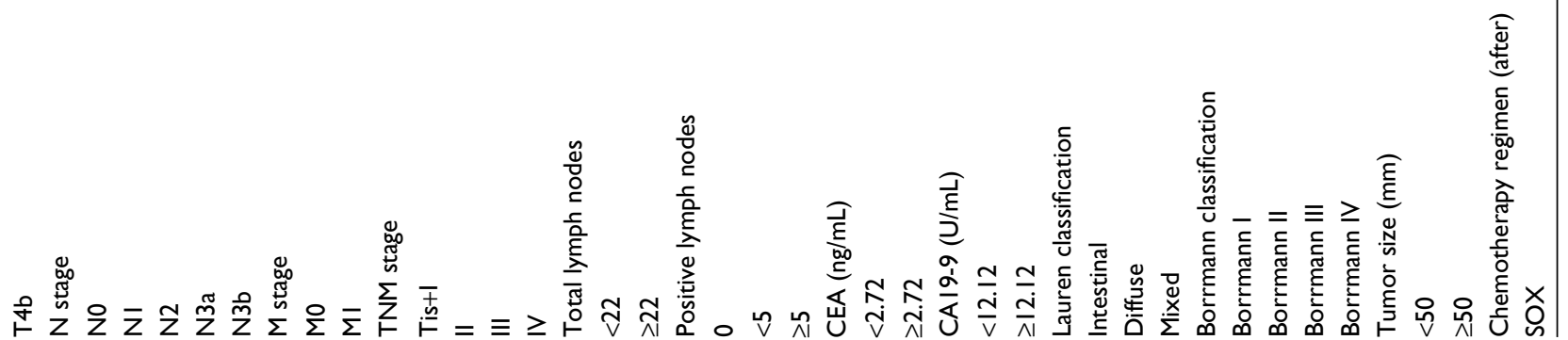




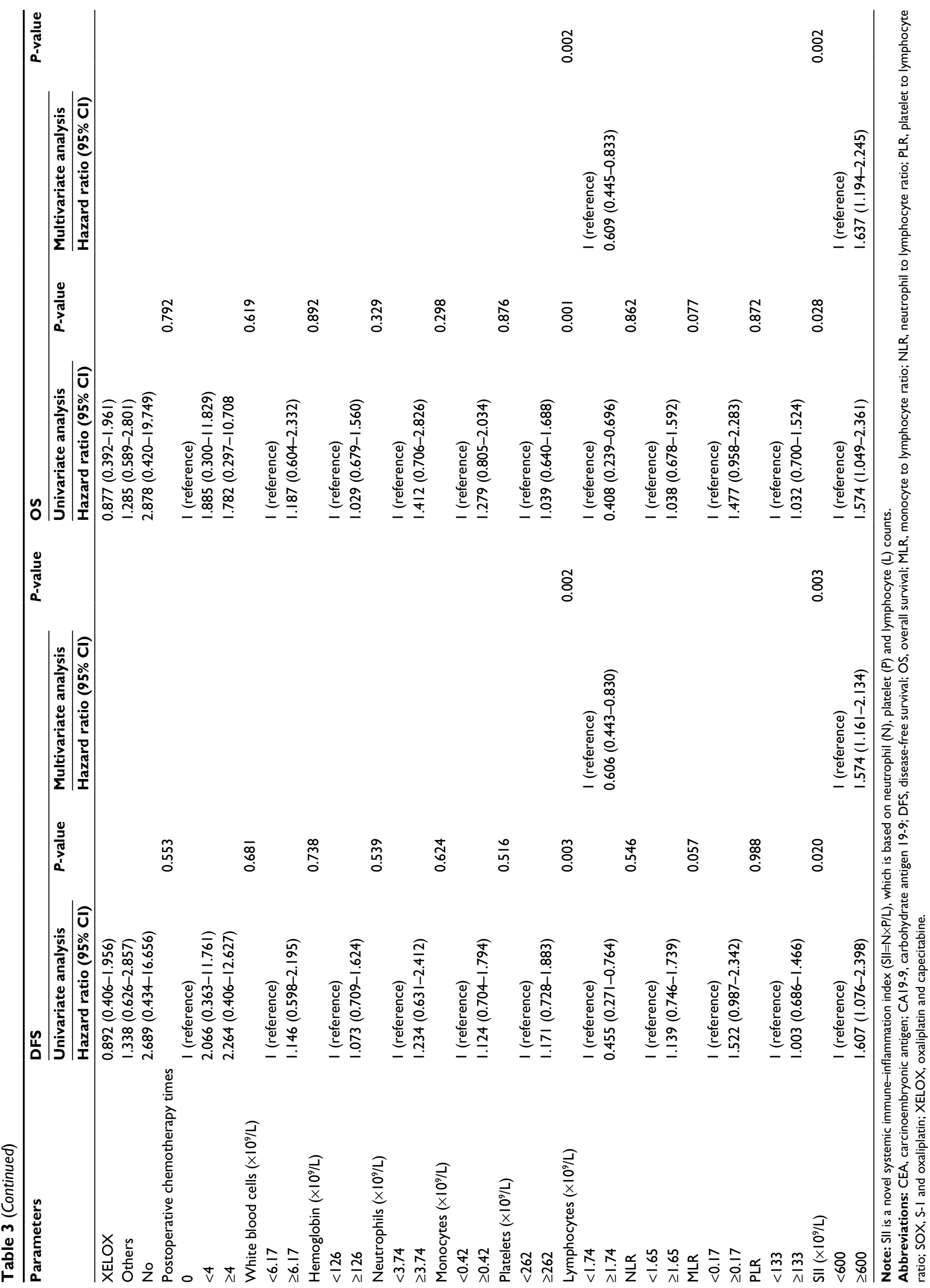


log-rank test, the mean DFS and OS time for patients with low SII were longer than in those with high SII $\left(\chi^{2}=4.572\right.$, $P=0.033$ and $\chi^{2}=4.593, P=0.032$, respectively; Figure 1E, F).

The SII was a significant prognostic factor with the cutoff value of $600 \times 10^{9} / \mathrm{L}$ on DFS and OS in the NCT group. In the NNCT group, the mean DFS and OS for patients with low SII were 57.29 months (range 1.93-111.97 months) and 63.25 months (range 3.03-117.37 months), respectively. The mean DFS and OS for patients with high SII were 46.50 months (range 1.03-99.50 months) and 51.18 months (range 3.10-104.10 months), respectively. By using log-rank test, the mean DFS and OS time for patients with low SII were longer than in those with high $\operatorname{SII}\left(\chi^{2}=0.636, P=0.425\right.$ and $\chi^{2}=1.046$, $P=0.306$, respectively; Figure $1 \mathrm{G}, \mathrm{H})$. However, the SII was not a significant prognostic factor using the cutoff value of $600 \times 10^{9} / \mathrm{L}$ on DFS and OS in the NNCT group.

\section{Survival and evaluation of the prognostic significance of SII}

For all patients we enrolled, the 1-, 3- and 5-year rates of DFS and OS in low SII were 78.1\% (132/169), 42.6\% (72/169) and $32.0 \%(54 / 169)$, and $88.2 \%$ (149/169), 47.9\% (81/169) and $34.3 \%$ (58/169), respectively. The 1-, 3- and 5-year rates of DFS and OS in high SII were 72.4\% (89/123), 31.7\% $(39 / 123)$ and $23.6 \%(29 / 123)$, and $81.3 \%$ (100/123), 35.0\% $(43 / 123)$ and $25.2 \%(31 / 123)$, respectively. In the NCT group, the 1-, 3- and 5-year rates of DFS and OS in low SII were $76.9 \%$ (40/52), $28.8 \%(15 / 52)$ and $11.5 \%(6 / 52)$, and $86.5 \%$ (45/52), 30.8\% (16/52) and 15.4\% (8/52), respectively. The 1-, 3- and 5-year rates of DFS and OS in high SII were $69.1 \%(38 / 55), 20.0 \%(11 / 55)$ and $7.3 \%(4 / 55)$, and $83.6 \%$
(46/55), $21.8 \%(12 / 55)$ and $9.1 \%(5 / 55)$, respectively. In the NNCT group, the 1-, 3- and 5-year rates of DFS and OS in low SII were $78.6 \%(92 / 117), 48.7 \%(57 / 117)$ and $41.0 \%$ (48/117), and 88.9\% (104/117), 55.6\% (65/117) and $42.7 \%$ (50/117), respectively. The 1-, 3- and 5-year rates of DFS and OS in high SII were $75.0 \%$ (51/68), 41.2\% (28/68) and $36.8 \%(25 / 68)$, and $79.4 \%(54 / 68), 45.6 \%$ (31/68) and $38.2 \%$ (26/68), respectively. Meanwhile, the patients with low SII had better 1-, 3- and 5-year rates of DFS and OS than those with high SII. Patients with gastric cancer who had lower SII were more likely to have longer DFS and OS (Table 4; Figure 2).

\section{Association of pathologic stage and SII in patients with gastric cancer}

In univariate and multivariate analyses, we found that pathologic $\mathrm{T}$ stage, pathologic $\mathrm{N}$ stage, pathologic $\mathrm{M}$ stage and pathologic TNM stage were the significant prognostic factors (Table 3). In order to further investigate the prognostic efficiency of SII, the SII was analyzed by the pathologic TNM stage. We defined the patients with pathologic Tis+I stage as early stage gastric cancer and the patients with pathologic II+III+IV stage as advanced stage gastric cancer, and we used the log-rank test to analyze the SII with different pathologic stages. For all patients, the results indicated that patients with low SII had longer DFS and OS than those with high SII in early stage gastric cancer $\left(\chi^{2}=0.107, P=0.744\right.$ and $\chi^{2}=0.356$, $P=0.551$, respectively; Figure 3A, B). Meanwhile, patients with low SII had longer DFS and OS than those with high SII in advanced stage gastric cancer $\left(\chi^{2}=4.139, P=0.042\right.$ and $\chi^{2}=5.052, P=0.025$, respectively; Figure $\left.3 \mathrm{C}, \mathrm{D}\right)$.

Table 4 I-, 3- and 5-year DFS and OS rates of patients with gastric cancer

\begin{tabular}{|c|c|c|c|c|c|c|c|}
\hline \multirow[t]{2}{*}{ Parameters } & \multirow[t]{2}{*}{ Cases (n) } & \multicolumn{3}{|l|}{ DFS } & \multicolumn{3}{|l|}{ OS } \\
\hline & & I year (\%) & 3 years (\%) & 5 years $(\%)$ & I year (\%) & 3 years (\%) & 5 years (\%) \\
\hline NCT+NNCT & 292 & & & & & & \\
\hline Low SII & 169 & I 32 (78.I) & $72(42.6)$ & $54(32.0)$ & 149 (88.2) & 81 (47.9) & $58(34.3)$ \\
\hline High SII & 123 & $89(72.4)$ & $39(31.7)$ & $29(23.6)$ & 100 (8I.3) & $43(35.0)$ & 31 (25.2) \\
\hline$\chi^{2}$ & & 1.278 & 3.587 & 2.455 & 2.672 & 4.901 & 2.792 \\
\hline$P$ value & & 0.258 & 0.058 & 0.117 & 0.102 & 0.027 & 0.095 \\
\hline NCT & 107 & & & & & & \\
\hline Low SII & 52 & 40 (76.9) & I5 (28.8) & 6 (II.5) & $45(86.5)$ & $16(30.8)$ & $8(15.4)$ \\
\hline High SII & 55 & $38(69.1)$ & II (20.0) & $4(7.3)$ & $46(83.6)$ & $12(21.8)$ & $5(9.1)$ \\
\hline$\chi^{2}$ & & 0.830 & 1.137 & 0.574 & 0.177 & 1.108 & 0.992 \\
\hline$P$ value & & 0.362 & 0.286 & 0.519 & 0.674 & 0.292 & 0.319 \\
\hline NNCT & 185 & & & & & & \\
\hline Low SII & 117 & $92(78.6)$ & 57 (48.7) & $48(4 I .0)$ & 104 (88.9) & $65(55.6)$ & $50(42.7)$ \\
\hline High SII & 68 & $51(75.0)$ & $28(4 \mid .2)$ & $25(36.8)$ & $54(79.4)$ & $31(45.6)$ & $26(38.2)$ \\
\hline$\chi^{2}$ & & 0.323 & 0.985 & 0.327 & 3.099 & I.7II & 0.360 \\
\hline$P$ value & & 0.570 & 0.321 & 0.568 & 0.078 & 0.191 & 0.549 \\
\hline
\end{tabular}

Note: SII is a novel systemic immune-inflammation index ( $\mathrm{SII}=\mathrm{N} \times \mathrm{P} / \mathrm{L})$, which is based on neutrophil $(\mathrm{N})$, platelet $(\mathrm{P})$ and lymphocyte $(\mathrm{L})$ counts.

Abbreviations: DFS, disease-free survival; NCT, neoadjuvant chemotherapy; NNCT, non-neoadjuvant chemotherapy; OS, overall survival. 

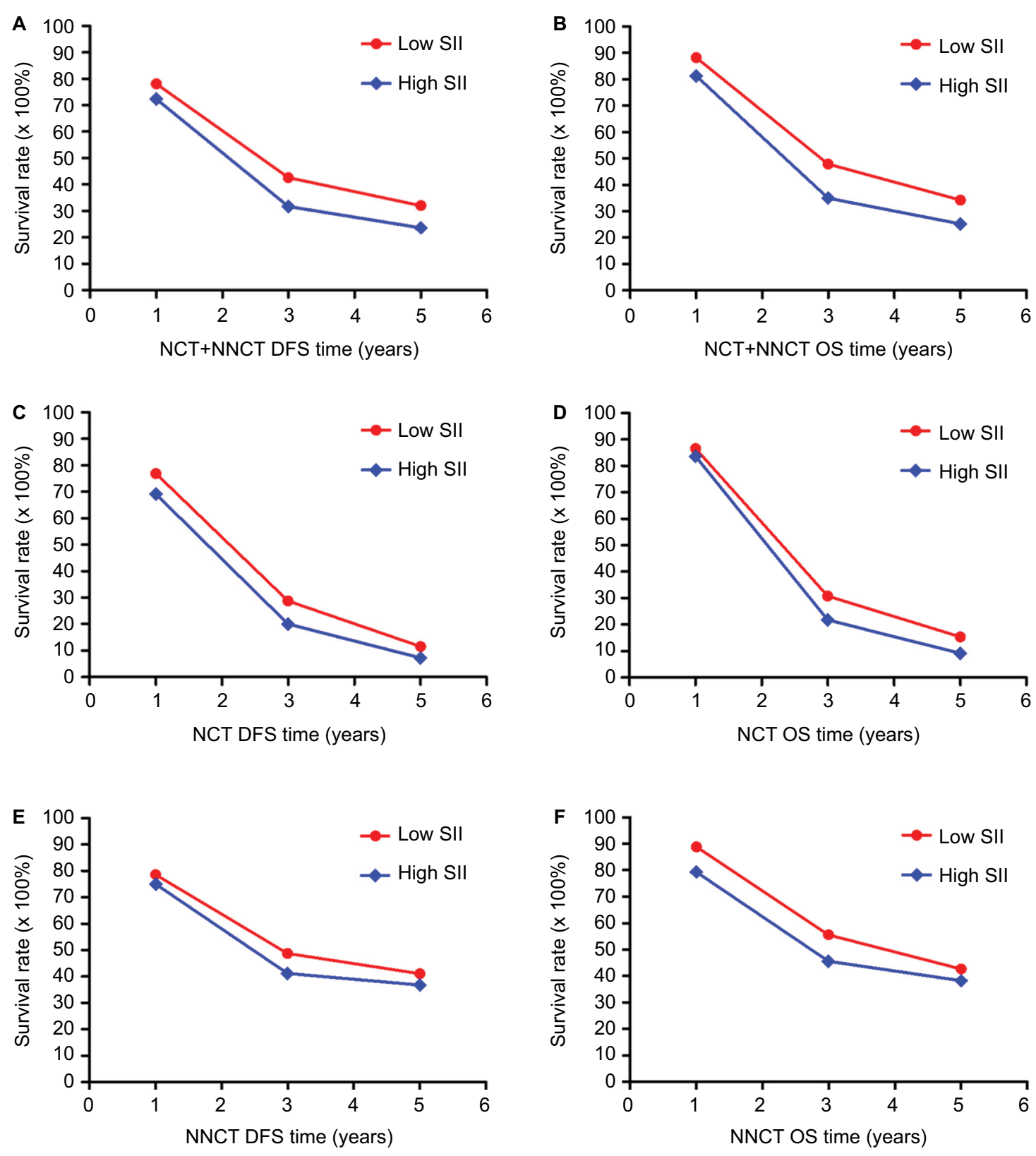

Figure 2 The 1-, 3- and 5-year rates of DFS and OS in patients with gastric cancer.

Notes: (A): The I-, 3- and 5-year rates of DFS in all patients with gastric cancer. (B) The I-, 3- and 5-year rates of OS in all patients with gastric cancer. (C) The I-, 3- and 5-year rates of DFS in the NCT group. (D) The I-, 3- and 5-year rates of OS in the NCT group. (E) The I-, 3- and 5-year rates of DFS in the NNCT group. (F) The I-, 3- and 5-year rates of OS in the NNCT group.

Abbreviations: DFS, disease-free survival; NCT, neoadjuvant chemotherapy; NNCT, non-neoadjuvant chemotherapy; OS, overall survival.

To further study SII in advanced stage gastric cancer, we reanalyzed the pathologic II stage, pathologic III stage and pathologic IV stage. We found that patients with low SII had longer DFS and OS than those with high SII in pathologic II stage $\left(\chi^{2}=0.852, P=0.356\right.$ and $\chi^{2}=1.036, P=0.309$, respectively; Figure $3 \mathrm{E}, \mathrm{F})$, pathologic III stage $\left(\chi^{2}=4.310\right.$, $P=0.038$ and $\chi^{2}=4.922, P=0.027$, respectively; Figure $\left.3 \mathrm{G}, \mathrm{H}\right)$ and pathologic IV stage $\left(\chi^{2}=6.616, P=0.010\right.$ and $\chi^{2}=2.696$, $P=0.101$, respectively; Figure 3I, J).

\section{Correlation between SII and neoadjuvant chemotherapy or postoperative chemotherapy with DFS and OS}

In the NCT group, all patients were treated with neoadjuvant chemotherapy, with the median value of preoperative chemotherapy times being 3 . Ninety-three patients were treated with postoperative chemotherapy, with the median value of postoperative chemotherapy times being 4 . The clinical 

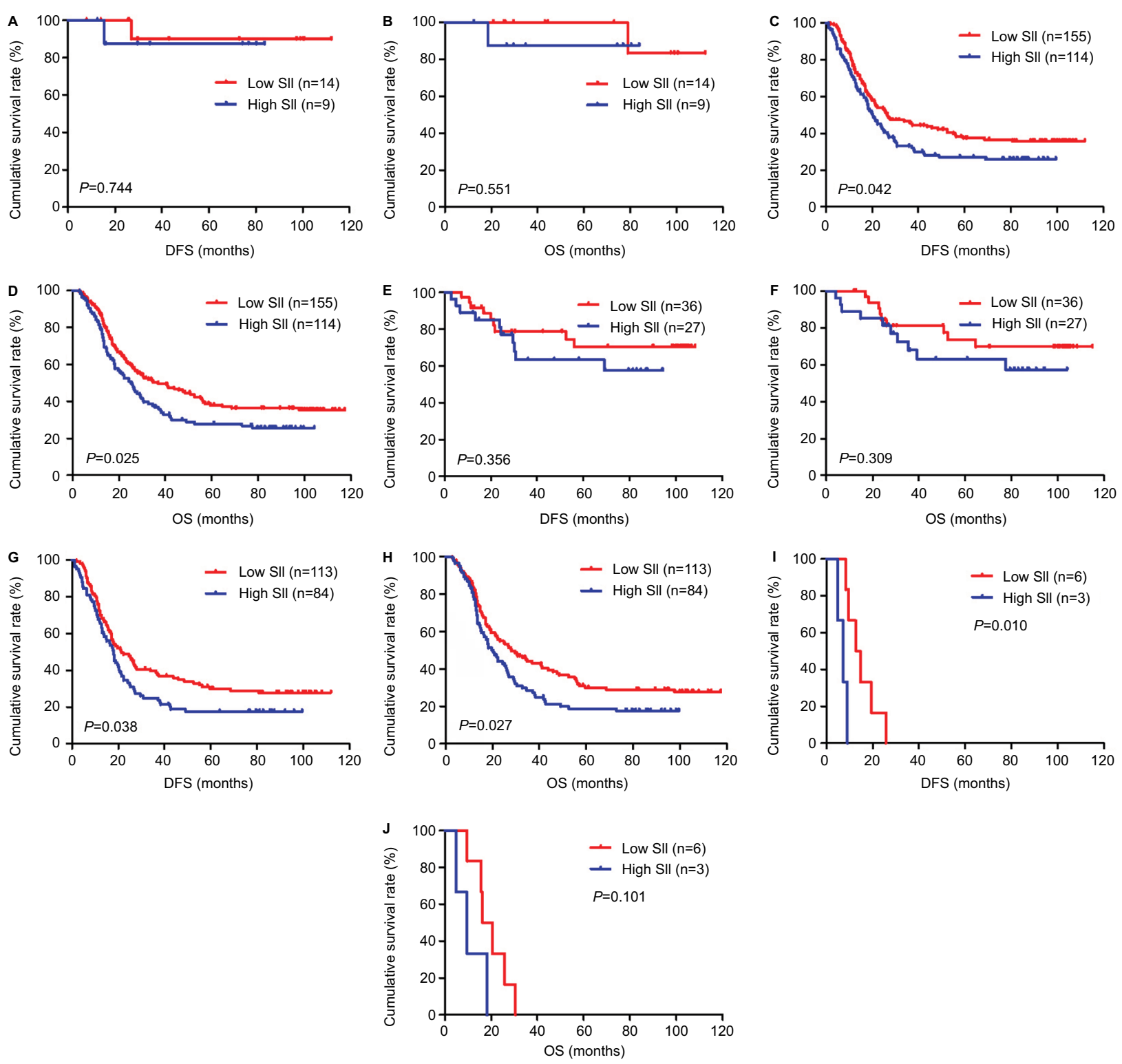

Figure 3 DFS and OS for the SII of patients with gastric cancer in pathologic stage.

Notes: (A) Kaplan-Meier analysis of DFS for the SII of patients with gastric cancer in early stage gastric cancer. (B) Kaplan-Meier analysis of OS for the SII of patients with gastric cancer in early stage gastric cancer. (C) Kaplan-Meier analysis of DFS for the SIl of patients with gastric cancer in advanced stage gastric cancer. (D) Kaplan-Meier analysis of OS for the SII of patients with gastric cancer in advanced stage gastric cancer. (E) Kaplan-Meier analysis of DFS for the SII of patients with gastric cancer in pathologic II stage. (F) Kaplan-Meier analysis of OS for the SII of patients with gastric cancer in pathologic II stage. (G) Kaplan-Meier analysis of DFS for the SII of patients with gastric cancer in pathologic III stage. (H) Kaplan-Meier analysis of OS for the SII of patients with gastric cancer in pathologic III stage. (I) Kaplan-Meier analysis of DFS for the SII of patients with gastric cancer in pathologic IV stage. (J) Kaplan-Meier analysis of OS for the SII of patients with gastric cancer in pathologic IV stage. SII is a novel systemic immune-inflammation index $(\mathrm{SII}=\mathrm{N} \times \mathrm{P} / \mathrm{L})$, which is based on neutrophil $(\mathrm{N})$, platelet $(\mathrm{P})$ and lymphocyte $(\mathrm{L})$ counts.

Abbreviations: DFS, disease-free survival; OS, overall survival.

objective response rate was $75.7 \%(81 / 107)$, and the clinical benefit rate was $82.2 \%(88 / 107)$. In the NNCT group, 135 patients were treated with postoperative chemotherapy, with the median value of postoperative chemotherapy times being 4. We found that SII was not associated with chemotherapy regimen and chemotherapy time (Table 5).

In the NCT group, we stratified patients undergoing neoadjuvant chemotherapy and postoperative chemotherapy as A group and those undergoing neoadjuvant chemotherapy but not postoperative chemotherapy as B group. We reana- lyzed A group and B group. The results indicated that the mean DFS and OS for patients in A group were 46.35 months (range 1.17-112.37 months) and 47.63 months (range 4.03-112.37 months), respectively. The mean DFS and OS for patients in B group were 30.21 months (range 4.90-96.00 months) and 30.68 months (range 4.90-96.00 months), respectively. We found that patients in A group had longer DFS and OS than those in B group $\left(\chi^{2}=1.456\right.$, $P=0.228$ and $\chi^{2}=2.289, P=0.130$, respectively; Figure 4A, B). The correlation between SII and neoadjuvant chemo- 
Table 5 Correlation between SII and neoadjuvant chemotherapy or postoperative chemotherapy of patients with gastric cancer

\begin{tabular}{|c|c|c|c|c|c|c|c|c|}
\hline \multirow[t]{2}{*}{ Parameters } & \multicolumn{3}{|l|}{ NCT } & \multirow[t]{2}{*}{$P$-value } & \multicolumn{3}{|l|}{ NNCT } & \multirow[t]{2}{*}{$P$-value } \\
\hline & Low SII $<600$ & High SII $\geq 600$ & $\chi^{2}$ & & Low SII $<600$ & High SII $\geq 600$ & $\chi^{2}$ & \\
\hline Cases (n) & 52 & 55 & & & 117 & 68 & & \\
\hline Chemotherapy regimen (before) & & & 0.442 & 0.802 & & & & \\
\hline sox & 20 & 15 & & & & & & \\
\hline XELOX & 26 & 30 & & & & & & \\
\hline Others & 6 & 10 & & & & & & \\
\hline Preoperative chemotherapy times & & & 1.636 & 0.201 & & & & \\
\hline$<3$ & 31 & 26 & & & & & & \\
\hline$\geq 3$ & 21 & 29 & & & & & & \\
\hline Chemotherapy regimen (after) & & & 2.348 & 0.503 & & & 0.976 & $0.807^{*}$ \\
\hline sox & 19 & 13 & & & 2 & I & & \\
\hline XELOX & 19 & 22 & & & 37 & 17 & & \\
\hline Others $^{a}$ & 8 & 12 & & & 54 & 34 & & \\
\hline No & 6 & 8 & & & 24 & 16 & & \\
\hline Postoperative chemotherapy times & & & 0.317 & 0.854 & & & 3.420 & 0.181 \\
\hline 0 & 6 & 8 & & & 24 & 16 & & \\
\hline$<4$ & 26 & 25 & & & 29 & 24 & & \\
\hline$\geq 4$ & 20 & 22 & & & 64 & 28 & & \\
\hline Response & & & 4.512 & $0.211 *$ & & & & \\
\hline $\mathrm{CR}$ & 7 & 2 & & & & & & \\
\hline PR & 35 & 37 & & & & & & \\
\hline SD & 2 & 5 & & & & & & \\
\hline PD & 8 & 11 & & & & & & \\
\hline
\end{tabular}

Notes: *Fisher's exact test. a Others include TS (paclitaxel and S-I), FOLFOX (folinic, oxaliplatin and fluorouracil), TCF (paclitaxel, cisplatin and fluorouracil), ECF (epirubicin, cisplatin and fluorouracil), TF (docetaxel and fluorouracil), DCF (docetaxel, cisplatin and fluorouracil) and other fluoropyrimidine-based adjuvant chemotherapy. SIl is a novel systemic immune-inflammation index $(\mathrm{SII}=\mathrm{N} \times \mathrm{P} / \mathrm{L})$, which is based on neutrophil $(\mathrm{N})$, platelet $(\mathrm{P})$ and lymphocyte $(\mathrm{L})$ counts.

Abbreviations: CR, complete response; NCT, neoadjuvant chemotherapy; NNCT, non-neoadjuvant chemotherapy; PD, progression of disease; PR, partial response; SD, stable disease; SOX, S-I and oxaliplatin; XELOX, oxaliplatin and capecitabine.
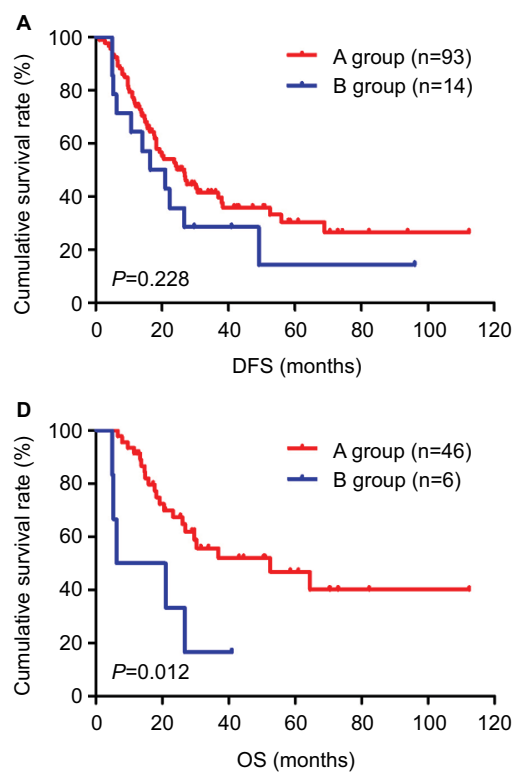
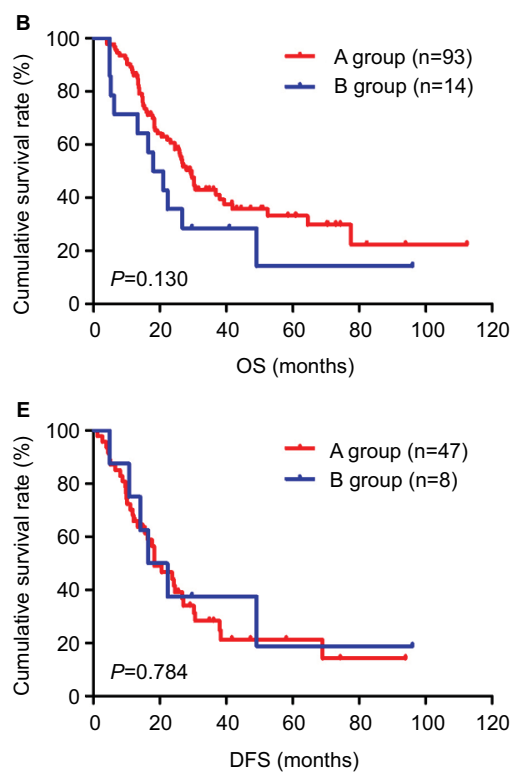
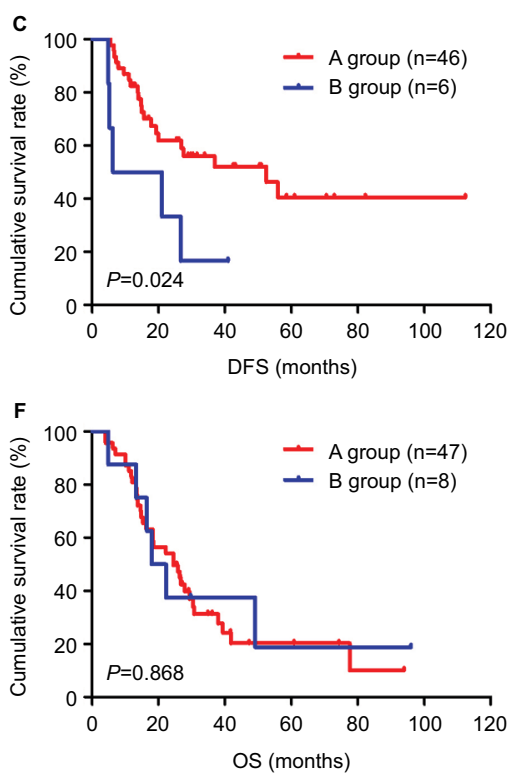

Figure 4 DFS and OS of patients with gastric cancer receiving neoadjuvant chemotherapy and postoperative chemotherapy in the NCT group.

Notes: Patients receiving neoadjuvant chemotherapy and postoperative chemotherapy form the A group and those receiving neoadjuvant chemotherapy but not postoperative chemotherapy form the B group. (A) Kaplan-Meier analysis of DFS for the SIl of patients with gastric cancer receiving neoadjuvant chemotherapy and postoperative chemotherapy. (B) Kaplan-Meier analysis of OS for the SII of patients with gastric cancer receiving neoadjuvant chemotherapy and postoperative chemotherapy. (C) KaplanMeier analysis of DFS for low SII of patients with gastric cancer receiving neoadjuvant chemotherapy and postoperative chemotherapy. (D) Kaplan-Meier analysis of OS for low SII of patients with gastric cancer receiving neoadjuvant chemotherapy and postoperative chemotherapy. (E) Kaplan-Meier analysis of DFS for high SII of patients with gastric cancer receiving neoadjuvant chemotherapy and postoperative chemotherapy. (F) Kaplan-Meier analysis of OS for high SII of patients with gastric cancer receiving neoadjuvant chemotherapy and postoperative chemotherapy. SII is a novel systemic immune-inflammation index $(\mathrm{SII}=\mathrm{N} \times \mathrm{P} / \mathrm{L})$, which is based on neutrophil $(\mathrm{N})$, platelet $(\mathrm{P})$ and lymphocyte (L) counts.

Abbreviations: DFS, disease-free survival; NCT, neoadjuvant chemotherapy; OS, overall survival. 
therapy was further evaluated, and, the SII was analyzed by the chemotherapy in A group and B group. For patients with low SII in the A group, the median DFS and OS were 59.69 months (range 5.63-112.37 months) and 61.73 months (range 6.60-112.37 months), respectively. The median DFS and OS for patients in the B group were 17.54 months (range 4.90-40.93 months) and 17.54 months (range 4.90-40.93 months), respectively. We found that patients with low SII in A group had longer DFS and OS than those in B group $\left(\chi^{2}=5.110, P=0.024\right.$ and $\chi^{2}=6.251, P=0.012$, respectively; Figure 4C, D). For patients with high SII in the A group, the median DFS and OS were 18.40 months (range 1.17-93.87 months) and 24.43 months (range 4.03-93.87 months), respectively. The median DFS and OS for patients in the B group were 16.50 months (range 4.93-96.00 months) and 17.93 months (range 4.93-96.00 months), respectively. We found that patients with high SII in A group had longer DFS and OS than those in B group $\left(\chi^{2}=0.075, P=0.784\right.$ and $\chi^{2}=0.028, P=0.868$, respectively; Figure $4 \mathrm{E}, \mathrm{F}$ ).

In the NNCT group, we stratified patients receiving postoperative chemotherapy as $\mathrm{C}$ group and those not receiving postoperative chemotherapy as $\mathrm{D}$ group. We reanalyzed $\mathrm{C}$ group and $\mathrm{D}$ group. The results indicated that the mean DFS and OS for patients in C group were 37.47 months (range 1.03-111.97 months) and 55.13 months (range 3.57-117.37 months), respectively. The results also indicated that the mean DFS and OS for patients in D group were 17.90 months (range 2.77-108.10 months) and 18.70 months (range 3.03-114.97 months), respectively. We found that patients in $\mathrm{C}$ group had longer DFS and OS than those in D group $\left(\chi^{2}=9.551, P=0.002\right.$ and $\chi^{2}=14.445, P<0.001$, respectively; Figure 5A, B). The correlation between SII and postoperative chemotherapy was further evaluated, and the SII was analyzed by the chemotherapy in $\mathrm{C}$ group and D group. For patients with low SII in C group, the mean DFS and OS were 52.50 months (range 1.93-111.97 months) and 56.07 months (range 3.57-117.37 months), respectively. The mean DFS and OS for patients in D group were 21.67 months (range 4.47-108.10 months) and 25.37 months (range 3.03-114.97 months), respectively. We found that patients with low SII in C group had longer DFS and OS than those in D group $\left(\chi^{2}=5.137, P=0.023\right.$ and $\chi^{2}=7.514, P=0.006$, respectively; Figure 5C, D). For patients with high SII in C group, the mean DFS and OS were 29.60 months (range 1.03-99.50 months) and 38.17 months (range 3.57-104.10 months), respectively. The mean DFS and OS for patients in D group were 26.93 months (range 2.77-92.13 months) and 28.08 months (range 3.10-98.33 months), respectively. We found that patients with high SII in C group had longer DFS and OS than those in $\mathrm{D}$ group $\left(\chi^{2}=4.310, P=0.038\right.$ and $\chi^{2}=6.521$, $P=0.011$, respectively; Figure 5E, F).
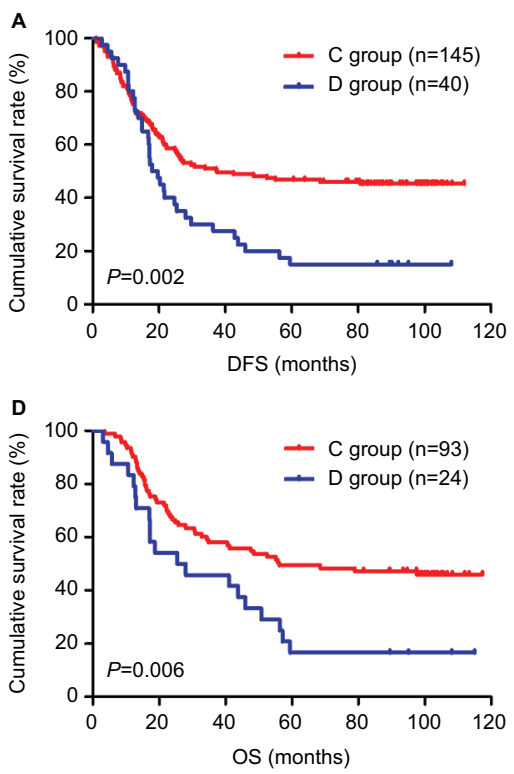
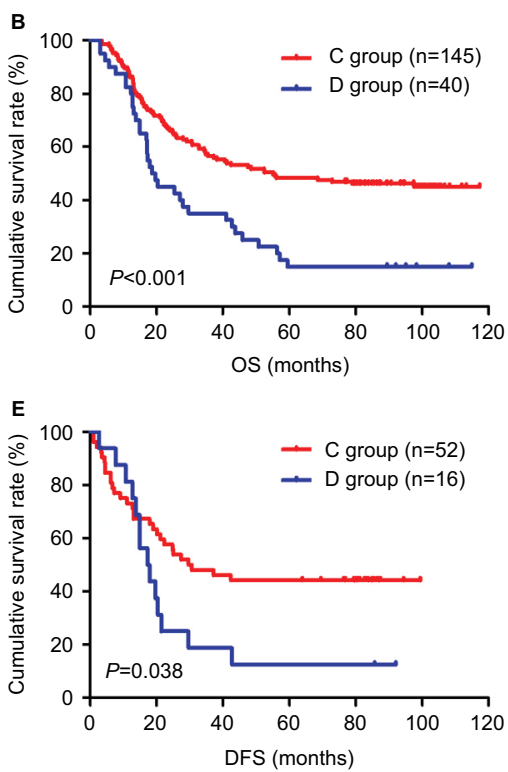
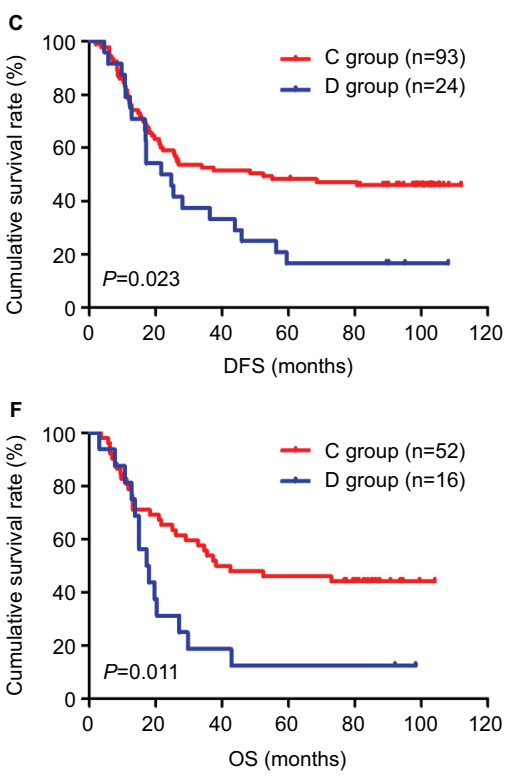

Figure 5 DFS and OS of patients with gastric cancer receiving postoperative chemotherapy in the NNCT group.

Notes: Patients receiving postoperative chemotherapy form the $C$ group and those not receiving postoperative chemotherapy form the $D$ group. (A) Kaplan-Meier analysis of DFS for the SII of patients with gastric cancer receiving postoperative chemotherapy. (B) Kaplan-Meier analysis of OS for the SII of patients with gastric cancer receiving postoperative chemotherapy. (C) Kaplan-Meier analysis of DFS for low SIl of patients with gastric cancer receiving postoperative chemotherapy. (D) Kaplan-Meier analysis of OS for low SII of patients with gastric cancer receiving postoperative chemotherapy. (E) Kaplan-Meier analysis of DFS for high SIl of patients with gastric cancer receiving postoperative chemotherapy. (F) Kaplan-Meier analysis of OS for high SII of patients with gastric cancer receiving postoperative chemotherapy. SII is a novel systemic immune-inflammation index ( $\mathrm{SIl}=\mathrm{N} \times \mathrm{P} / \mathrm{L})$, which is based on neutrophil $(\mathrm{N})$, platelet $(\mathrm{P})$ and lymphocyte $(\mathrm{L})$ counts.

Abbreviations: DFS, disease-free survival; NNCT, non-neoadjuvant chemotherapy; OS, overall survival. 


\section{Correlation between SII and toxicity}

\section{assessment}

In the NCT group, we evaluated and analyzed the toxicities after administering neoadjuvant chemotherapy for two cycles. We found that the most common toxicities after neoadjuvant chemotherapy were hematologic, and most of these toxicities were mild. The results by the National Cancer Institute Common Toxicity Criteria grade indicated that grade $1 / 2$ anemia, leukopenia, neutropenia and thrombocytopenia were recorded in 30.8\% (33/107), 21.5\% (23/107), 26.2\% (28/107) and $12.1 \%$ (13/107), respectively; grade $3 / 4$ anemia, leukopenia, neutropenia and thrombocytopenia were recorded in $2.8 \%(3 / 107), 0.0 \%(0 / 107), 1.8 \%(2 / 107)$ and $0.0 \%(0 / 107)$, respectively (Table 6). Myelosuppression and gastrointestinal reaction toxicities were also recorded in 49.5\% (53/107) and $83.2 \%(89 / 107)$, respectively (Table 6$)$. There were no chemotherapy-related deaths in this study. Further studies on toxicity assessment in SII showed that there were no differences using the cutoff value $600 \times 10^{9} / \mathrm{L}$ of SII in anemia, neutropenia, thrombocytopenia, myelosuppression and gastrointestinal reaction $\left(\chi^{2}=5.061,5.687,0.992,0.009\right.$ and 0.817 , $P>0.05)$, except in leukopenia $\left(\chi^{2}=5.156, P<0.05\right.$; Table 6$)$.

Table 6 Main toxicities according to $\mathrm{NCl}-\mathrm{CTC}$ scale of the patients with gastric cancer undergoing neoadjuvant chemotherapy

\begin{tabular}{|c|c|c|c|c|c|}
\hline \multirow[t]{2}{*}{ Toxicity } & & \multicolumn{3}{|c|}{ NCT group } & \multirow[t]{2}{*}{$P$-value } \\
\hline & & $\begin{array}{l}\text { Low SII } \\
<600\end{array}$ & $\begin{array}{l}\text { High SII } \\
\geq 600\end{array}$ & $\chi^{2}$ & \\
\hline Cases (n) & 107 & 52 & 55 & & \\
\hline \multicolumn{6}{|l|}{ Anemia } \\
\hline Grade 0 & 7I (66.4\%) & 40 (76.9\%) & 31 (56.4\%) & 5.061 & 0.080 \\
\hline Grade I-2 & $33(30.8 \%)$ & II (2I.2\%) & $22(40.0 \%)$ & & \\
\hline Grade 3-4 & $3(2.8 \%)$ & I (I.9\%) & $2(3.6 \%)$ & & \\
\hline \multicolumn{6}{|l|}{ Leukopenia } \\
\hline Grade 0 & $84(78.5 \%)$ & $36(69.2 \%)$ & 48 (87.3\%) & 5.156 & 0.023 \\
\hline Grade I-2 & $23(21.5 \%)$ & $16(30.8 \%)$ & 7 (I2.7\%) & & \\
\hline Grade 3-4 & $0(0.0 \%)$ & $0(0.0 \%)$ & $0(0.0 \%)$ & & \\
\hline \multicolumn{6}{|c|}{ Neutropenia } \\
\hline Grade 0 & 77 (72.0\%) & 32 (61.5\%) & 45 (81.8\%) & 5.687 & 0.058 \\
\hline Grade I-2 & $28(26.2 \%)$ & $19(36.5 \%)$ & $9(16.4 \%)$ & & \\
\hline Grade 3-4 & $2(1.8 \%)$ & I (2.0\%) & $\mathrm{I}(\mathrm{I} .8 \%)$ & & \\
\hline \multicolumn{6}{|c|}{ Thrombocytopenia } \\
\hline Grade 0 & $94(87.9 \%)$ & $44(84.6 \%)$ & $50(90.9 \%)$ & 0.992 & 0.319 \\
\hline Grade I-2 & $13(12.1 \%)$ & $8(15.4 \%)$ & $5(9.1 \%)$ & & \\
\hline Grade 3-4 & $0(0.0 \%)$ & $0(0.0 \%)$ & $0(0.0 \%)$ & & \\
\hline \multicolumn{6}{|c|}{ Myelosuppression } \\
\hline Yes & $53(49.5 \%)$ & $26(50.0 \%)$ & 27 (49.1\%) & 0.009 & 0.925 \\
\hline No & $54(50.5 \%)$ & $26(50.0 \%)$ & $28(50.9 \%)$ & & \\
\hline \multicolumn{6}{|c|}{ Gastrointestinal reaction } \\
\hline Yes & 89 (83.2\%) & 45 (86.5\%) & 44 (80.0\%) & 0.817 & 0.366 \\
\hline No & $18(16.8 \%)$ & 7 (I3.5\%) & II (20.0\%) & & \\
\hline
\end{tabular}

Note: SII is a novel systemic immune-inflammation index $(\mathrm{SII}=\mathrm{N} \times \mathrm{P} / \mathrm{L})$, which is based on neutrophil $(\mathrm{N})$, platelet $(\mathrm{P})$ and lymphocyte $(\mathrm{L})$ counts.

Abbreviations: $\mathrm{NCl}-\mathrm{CTC}$, National Cancer Institute Common Toxicity Criteria; NCT, neoadjuvant chemotherapy.

\section{Discussion}

With the rapid advances in surgical techniques and multimodal therapy, including radiotherapy, adjuvant chemotherapy, neoadjuvant chemotherapy, perioperative chemotherapy and targeted therapy, the clinical outcomes and quality of life have greatly improved for patients with advanced gastric cancer. ${ }^{25}$ Nowadays, neoadjuvant chemotherapy is in the limelight, with decrease in postoperative complications, morbidity and mortality, and its efficacy and safety being its advantages. ${ }^{35}$ Gastric carcinoma is one of the diseases with the highest tumor burden, as measured by disabilityadjusted life-years. ${ }^{26}$ Therefore, for the sake of choosing the optimal treatment regimen and improving clinical outcomes in patients with gastric cancer, precise predictors that identify those patients who are more likely to benefit from neoadjuvant chemotherapy are needed.

The tumor cells influence the proinflammatory mediators, and systemic inflammation promotes tumor cell proliferation, migration and invasion by inhibiting apoptosis and promoting angiogenesis. ${ }^{27,28}$ Various studies have suggested that systemic immune and inflammatory cells, such as neutrophils, monocytes, platelets and lymphocytes, are associated with prognostic value in many malignancies. ${ }^{29-32}$ Moreover, several inflammation and immune-based prognostic indexes have been used to predict tumor recurrence and metastasis as prognostic factors, such as NLR, MLR and PLR. ${ }^{33-35}$ However, these predictors are usually based on one or two inflammatory cells, and their prediction ability for tumors may not be the best. Currently, SII based on neutrophil, platelet and lymphocyte counts, which can comprehensively reflect the balance of host immune and inflammatory status, has been developed to be an independent predictor of clinical outcomes of cancer in clinical practice ${ }^{36}$ Nevertheless, the mechanisms are still ambiguous and poorly understood.

Cancer and inflammation are closely connected, and several potential mechanisms may be used to explain that high SII is associated with poor survival. The tumor microenvironment, as well as the tumor cells inhabiting inflammatory cells, are very important in carcinogenesis and angiogenesis. Peripheral venous neutrophil is an indicator of acute and chronic inflammation and inhibits the immune system to promote tumor development, progression and metastasis via restraining the cytolytic activity of immune cells and secreting cytokines and chemokines, such as matrix metalloproteinase- 9 and vascular endothelial growth factor. ${ }^{37-39}$ The neutrophils also can promote adhesion between circulating tumor cells (CTCs) and metastatic target organ. Platelets can shield CTCs from immune attack and destruction by 
activated platelets, which in turn protect the CTCs from shearing stresses during circulation and promote the CTCs' epithelial mesenchymal transition and enhance their transendothelial migration and metastasis by releasing adenine nucleotides and opening endothelial barrier. ${ }^{40-42}$ Platelets also can promote increasing angiogenesis via the cytokine vascular endothelial growth factor. ${ }^{43}$ The lymphocytes are known to play a critical role in controlling tumor growth and progression by secreting cytokines, such as tumor necrosis factor- $\alpha$ and interferon- $\gamma$, and as well as suppressing tumor cell proliferation and migration. ${ }^{44-46}$ The downregulation of peripheral lymphocyte counts and decreased function can weaken host's anticancer immunity and impair cancer immune surveillance. ${ }^{47}$ Therefore, combined with these findings, the SII is considered a better objective indicator that reflects the balance of host inflammatory and immune status than other inflammation and immune-based prognostic indexes such as NLR, MLR and PLR.

In this study, the demographic and clinicopathologic characteristics of the 292 patients enrolled in the study were analyzed. We found that low SII was significantly associated with CEA in the NCT group, and gender, pathologic N stage, CA19-9 and tumor size in the NNCT group. Moreover, we analyzed the blood parameters in the two groups, and the results indicated that low SII was significantly associated with white blood cells, $\mathrm{Hb}$, neutrophils, platelets, as well as NLR, MLR and PLR. However, low SII was significantly associated with lymphocytes in the NNCT group and not associated with monocytes in the NCT and NNCT groups. In univariate and multivariate Cox regression analyses, radical resection, pathologic stage ( $\mathrm{T}, \mathrm{N}, \mathrm{M}$ and TNM stage), total lymph nodes, positive lymph nodes, Borrmann classification, lymphocytes and SII were the significant prognostic factors predicting improved DFS and OS.

The results demonstrated that SII had prognostic significance on using the cutoff value of $600 \times 10^{9} / \mathrm{L}$ on DFS and OS, and the mean DFS and OS time for patients with low SII were longer than for those with high SII. Although the mean DFS and OS time for patients with low SII were longer than for those with high SII in the NCT and NNCT groups, SII was a significant prognostic factor in the NCT group but not in the NNCT group. Meanwhile, the results also indicated that patients with gastric cancer who had lower SII were more likely to have longer 1-, 3- and 5-year rates of DFS and OS. Apart from these analysis, we also observed that patients with low SII had longer DFS and OS than those with high SII in early stage gastric cancer and advanced stage gastric cancer.
The correlations between SII and neoadjuvant chemotherapy and postoperative chemotherapy with DFS and OS were studied. We found that SII was not associated with chemotherapy regimen and chemotherapy time. All patients could tolerate the neoadjuvant chemotherapy toxicities, and the regimens were safe and effective. The most common toxicities after neoadjuvant chemotherapy were hematologic. The results indicated that in toxicity assessment, there was no difference in these toxicities using the cutoff value of $600 \times 10^{9} / \mathrm{L}$ for SII, except in leukopenia.

In summary, results of this study indicated that low SII would have better clinical outcomes with adjuvant chemotherapy, especially in receiving neoadjuvant chemotherapy. It is important to take into consideration the high gastric cancer morbidity and unbalanced medical condition in China, and that noninvasive, inexpensive and reproducible biomarkers should be used for the prevention and treatment of gastric cancer. A comprehensive understanding of hematologic parameters may help find new targets for subjective treatment and provide critical information for doctors to administer effective therapy in clinical practice.

However, there are several limitations that cannot be neglected in this study. First, this was a retrospective singlecenter study and the sample size was small. More patients with gastric cancer treated with neoadjuvant chemotherapy should be enrolled and a multicenter study should be conducted. Second, SII before surgery in patients with neoadjuvant chemotherapy was not analyzed, and further study should analyze and compare with SII before neoadjuvant chemotherapy. Third, the cutoff values of SII in different studies may be different because of the cumulative number of patients and the disease stage. Further prospective and well-designed randomized controlled trials are required to determine whether the cutoff value of $600 \times 10^{9} / \mathrm{L}$ of SII is correct, before it is adopted in routine practice.

\section{Conclusion}

SII may qualify as a noninvasive, cost-effective, convenient and reproducible prognostic indicator for patients with advanced gastric cancer undergoing neoadjuvant chemotherapy. Also, low SII can potentially be used to help clinicians identify those patients who will benefit from neoadjuvant chemotherapy. However, more studies are needed to verify the changes in systemic immune-inflammation index in larger groups of patients with advanced gastric cancer.

\section{Disclosure}

The authors report no conflicts of interest in this work. 


\section{References}

1. Siegel RL, Miller KD, Jemal A. Cancer Statistics, 2015. CA Cancer J Clin. 2015;65(1):5-29.

2. DeSantis CE, Lin CC, Mariotto AB, et al. Cancer treatment and survivorship statistics, 2014. CA Cancer J Clin. 2014;64(4):252-271.

3. Fock KM. Review article: the epidemiology and prevention of gastric cancer. Aliment Pharmacol Ther. 2014;40(3):250-260.

4. Ferro A, Peleteiro B, Malvezzi M, et al. Worldwide trends in gastric cancer mortality (1980-2011), with predictions to 2015, and incidence by subtype. Eur J Cancer. 2014;50(7):1330-1344.

5. Mackenzie M, SpithoffK, Jonker D. Systemic therapy for advanced gastric cancer: a clinical practice guideline. Curr Oncol. 2011;18(4):e202-e209.

6. Biondi A, Lirosi MC, D'Ugo D, et al. Neo-adjuvant chemo(radio) therapy in gastric cancer: current status and future perspectives. World $J$ Gastrointest Oncol. 2015;7(12):389-400.

7. Manoharan V, Lee S, Chong S, et al. Serial imaging using [18F] Fluorodeoxyglucose positron emission tomography and histopathologic assessment in predicting survival in a population of surgically resectable distal oesophageal and gastric adenocarcinoma following neoadjuvant therapy. Ann Nucl Med. 2017;31(4):315-323.

8. Mingol F, Gallego J, Orduña A, et al. Tumor regression and survival after perioperative MAGIC-style chemotherapy in carcinoma of the stomach and gastroesophageal junction. BMC Surg. 2015;15:66.

9. Neves Filho EH, de Sant'Ana RO, Nunes LV, Pires AP, da Cunha MD. Histopathological regression of gastric adenocarcinoma after neoadjuvant therapy: a critical review. APMIS. 2017;125(2):79-84.

10. Park SC, Chun HJ. Chemotherapy for advanced gastric cancer: review and update of current practices. Gut Liver. 2013;7(4):385-393.

11. el Aziz LM. Blood neutrophil-lymphocyte ratio predicts survival in locally advanced cancer stomach treated with neoadjuvant chemotherapy FOLFOX4. Med Oncol. 2014;31(12):311.

12. Kim KH, Kwon HC, Oh SY, et al. Clinicopathological significance of ERCC1, thymidylate synthase and glutathione S-transferase P1 expression for advanced gastric cancer patients receiving adjuvant 5-Fu and cisplatin chemotherapy. Biomarkers. 2011;16(1):74-82.

13. Lim H, Moon A. Inflammatory fibroblasts in cancer. Arch Pharm Res. 2016;39(8):1021-1031.

14. Elinav E, Nowarski R, Thaiss CA, Hu B, Jin C, Flavell RA. Inflammation-induced cancer: crosstalk between tumors, immune cells and microorganisms. Nat Rev Cancer. 2013;13(11):759-771.

15. Diakos CI, Charles KA, McMillan DC, Clarke SJ. Cancer-related inflammation and treatment effectiveness. Lancet Oncol. 2014;15(11):e493-e503.

16. Borsig L, Wolf MJ, Roblek M, Lorentzen A, Heikenwalder M. Inflammatory chemokines and metastasis-tracing the accessory. Oncogene. 2014;33(25):3217-3224.

17. Lolli C, Caffo O, Scarpi E, et al. Systemic immune-inflammation index predicts the clinical outcome in patients with mCRPC treated with Abiraterone. Front Pharmacol. 2016;7:376.

18. Lolli C, Basso U, Derosa L, et al. Systemic immune inflammation index predicts the clinical outcome in patients with metastatic renal cell cancer treated with sunitinib. Oncotarget. 2016;7(34):54564-54571.

19. Wang L, Wang C, Wang J, Huang X, Cheng Y. A novel systemic immune-inflammation index predicts survival and quality of life of patients after curative resection for esophageal squamous cell carcinoma. J Cancer Res Clin Oncol. 2017;143(10):2077-2086.

20. Amin MB, Amin MB, Greene FL, et al. The eighth edition AJCC cancer staging manual: continuing to build a bridge from a population-based to a more "personalized" approach to cancer staging. CA Cancer J Clin. 2017;67(2):93-99.

21. Lauren P. The two histological main types of gastric carcinoma: diffuse and so-called intestinaltype carcinoma. An attempt at a histo-clinical classification. Acta Pathol Microbiol Scand. 1965;64:31-49.

22. Cancer Genome Atlas Research Network. Comprehensive molecular characterization of gastric adenocarcinoma. Nature. 2014;513(7517):s202-s209.

23. Eisenhauer EA, Therasse P, Bogaerts J, et al. New response evaluation criteria in solid tumors: revised RESIST guideline (version 1.1). Eur J Cancer. 2009;45(2):228-247.
24. Caussanel JP, Lévi F, Brienza S, et al. Phase I trial of 5-day continuous venous infusion of oxaliplatin at circadian rhythmmodulated rate compared with constant rate. J Natl Cancer Inst. 1990;82(12):1046-1050.

25. Laurent Quéro, Sophie Guillerm, Christophe Hennequin. Neoadjuvant or adjuvant therapy for gastric cancer. World J Gastrointest Oncol. 2015;7(8):102-110.

26. Soerjomataram I, Lortet-Tieulent J, Parkin DM, et al. Global burden of cancer in 2008: a systematic analysis of disability-adjusted life-years in 12 world regions. Lancet. 2012;380(9856):1840-1850.

27. Mantovani A, Allavena P, Sica A, Balkwill F. Cancer-related inflammation. Nature. 2008;454(7203):436-444.

28. McMillan DC. Systemic inflammation, nutritional status and survival in patients with cancer. Curr Opin Clin Nutr Metab Care. 2009;12(3):223-226.

29. Li TJ, Jiang YM, Hu YF, et al. Interleukin-17-producing neutrophils link inflammation stimuli to disease progression by promoting angiogenesis in gastric cancer. Clin Cancer Res. 2017;23(6):1575-1585.

30. Eo WK, Jeong DW, Chang HJ, et al. Absolute monocyte and lymphocyte count prognostic score for patients with gastric cancer. World $J$ Gastroenterol. 2015;21(9):2668-2676.

31. Kılınçalp S, Ekiz F, Başar O, et al. Mean platelet volume could be possible biomarker in early diagnosis and monitoring of gastric cancer. Platelets. 2014;25(8):592-594.

32. Quigley DA, Kristensen V. Predicting prognosis and therapeutic response from interactions between lymphocytes and tumor cells. $\mathrm{Mol}$ Oncol. 2015;9(10):2054-2062.

33. Ock CY, Nam AR, Lee J, et al. Prognostic implication of antitumor immunity measured by the neutrophil-lymphocyte ratio and serum cytokines and angiogenic factors in gastric cancer. Gastric Cancer. 2017;20(2):254-262.

34. Chen L, Hao Y, Zhu LH, et al. Monocyte to lymphocyte ratio predicts survival in patients with advanced gastric cancer undergoing neoadjuvant chemotherapy. Onco Targets Ther. 2017;10:4007-4016.

35. Koh CH, Bhoo-Pathy N, Ng KL, et al. Utility of pre-treatment neutrophil-lymphocyte ratio and platelet-lymphocyte ratio as prognostic factors in breast cancer. Br J Cancer. 2015;113(1):150-158.

36. Feng JF, Chen S, Yang X. Systemic immune-inflammation index (SII) is a useful prognostic indicator for patients with squamous cell carcinoma of the esophagus. Medicine (Baltimore). 2017;96(4):e5886.

37. Kolaczkowska E, Kubes P. Neutrophil recruitment and function in health and inflammation. Nat Rev Immunol. 2013;13(3):159-175.

38. Tan KW, Chong SZ, Wong FH, et al. Neutrophils contribute to inflammatory lymphangiogenesis by increasing VEGF-A bioavailability and secreting VEGF-D. Blood. 2013;122(22):3666-3677.

39. Bausch D, Pausch T, Krauss T, et al. Neutrophil granulocyte derived MMP-9 is a VEGF independent functional component of the angiogenic switch in pancreatic ductal adenocarcinoma. Angiogenesis. 2011;14(3): 235-243.

40. Jenne CN, Urrutia R, Kubes P. Platelets: bridging hemostasis, inflammation, and immunity. Int J Lab Hematol. 2013;35(3):254-261.

41. Labelle M, Begum S, Hynes RO. Platelets guide the formation of early metastatic niches. Proc Natl Acad Sci USA. 2014;111(30):E3053-E3061.

42. Bambace NM, Holmes CE. The platelet contribution to cancer progression. J Thromb Haemost. 2011;9(2):237-249.

43. Benoy I, Salgado R, Colpaert C, Weytjens R, Vermeulen PB, Dirix LY. Serum interleukin 6, plasma VEGF, serum VEGF, and VEGF platelet load in breast cancer patients. Clin Breast Cancer. 2002;2(4):311-315.

44. Ferrone C, Dranoff G. Dual roles for immunity in gastrointestinal cancers. J Clin Oncol. 2010;28(26):4045-4051.

45. Fondevila C, Metges JP, Fuster J, et al. p53 and VEGF expression are independent predictors of tumor recurrence and survival following curative resection of gastric cancer. Br J Cancer. 2004;90(1):206-215.

46. Ogiya R, Niikura N, Kumaki N, et al. Comparison of tumor-infiltrating lymphocytes between primary and metastatic tumors in breast cancer patients. Cancer Sci. 2016;107(12):1730-1735.

47. Milne K, Alexander C, Webb JR, et al. Absolute lymphocyte count is associated with survival in ovarian cancer independent of tumorinfiltrating lymphocytes. J Transl Med. 2012;10:s33. 
Cancer Management and Research

\section{Publish your work in this journal}

Cancer Management and Research is an international, peer-reviewed open access journal focusing on cancer research and the optimal use of preventative and integrated treatment interventions to achieve improved outcomes, enhanced survival and quality of life for the cancer patient

The manuscript management system is completely online and includes

Submit your manuscript here: https://www.dovepress.com/cancer-management-and-research-journal 